\title{
Quasars Probing Quasars. X. The Quasar Pair Spectral Database
}

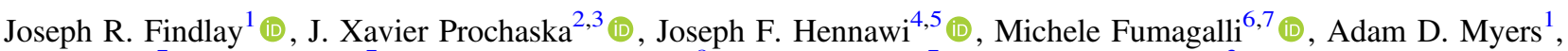 \\ Stephanie Bartle $^{7}$, Ben Chehade ${ }^{7}$, Michael A. DiPompeo ${ }^{8}$ (D), Tom Shanks ${ }^{7}$, Marie Wingyee Lau ${ }^{3}$ (D), and Kate H. R. Rubin ${ }^{9}$ (D) \\ ${ }^{1}$ University of Wyoming, Physics \& Astronomy 1000 E. University, Dept 3905 Laramie, WY 82071, USA \\ 2 Department of Astronomy and Astrophysics, University of California, 1156 High Street, Santa Cruz, California 95064, USA \\ ${ }^{3}$ University of California Observatories, Lick Observatory, 1156 High Street, Santa Cruz, California 95064, USA \\ ${ }^{4}$ Department of Physics, Broida Hall, University of California, Santa Barbara, CA 93106-9530, USA \\ ${ }^{5}$ Max-Planck-Institut für Astronomie, Königstuhl 17, D-69117 Heidelberg, Germany \\ ${ }^{6}$ Institute for Computational Cosmology, Durham University, South Road, Durham, DH1 3LE, UK \\ ${ }^{7}$ Centre for Extragalactic Astronomy, Department of Physics, Durham University, South Road, Durham, DH1 3LE, UK \\ ${ }^{8}$ Department of Physics and Astronomy, Dartmouth College, 6127 Wilder Laboratory, Hanover, NH 03755, USA \\ ${ }^{9}$ San Diego State University, Department of Astronomy, San Diego, CA 92182, USA \\ Received 2018 February 2; accepted 2018 March 31; published 2018 June 5
}

\begin{abstract}
The rare close projection of two quasars on the sky provides the opportunity to study the host galaxy environment of a foreground quasar in absorption against the continuum emission of a background quasar. For over a decade the "Quasars probing quasars" series has utilized this technique to further the understanding of galaxy formation and evolution in the presence of a quasar at $z>2$, resolving scales as small as a galactic disk and from bound gas in the circumgalactic medium to the diffuse environs of intergalactic space. Presented here is the public release of the quasar pair spectral database utilized in these studies. In addition to projected pairs at $z>2$, the database also includes quasar pair members at $z<2$, gravitational lens candidates, and quasars closely separated in redshift that are useful for small-scale clustering studies. In total, the database catalogs 5627 distinct objects, with 4083 lying within $5^{\prime}$ of at least one other source. A spectral library contains 3582 optical and near-infrared spectra for 3028 of the cataloged sources. As well as reporting on 54 newly discovered quasar pairs, we outline the key contributions made by this series over the last 10 years, summarize the imaging and spectroscopic data used for target selection, discuss the target selection methodologies, describe the database content, and explore some avenues for future work. Full documentation for the spectral database, including download instructions, is supplied at http://specdb. readthedocs.io/en/latest/.
\end{abstract}

Key words: catalogs - galaxies: evolution - galaxies: halos - quasars: absorption lines - quasars: general - surveys

\section{Introduction}

An understanding of the processes by which galaxies accrete, expel, and recycle gas is as essential to a complete theory of galaxy evolution as population demographics and star formation. At its largest scales, the universe is seen as a living network of filaments blossoming with clusters and galaxies at their intersections. This "cosmic web" contains $90 \%$ of all the baryonic material in the universe and supplies the fuel for galaxy formation along its filaments. The journey of this gas into the interstellar medium (ISM) occurs through the circumgalactic medium (CGM), a gravitationally bound gas reservoir, distinct from the ISM but contained within a region similar in extent to a galaxy's own virial radius. In their recent review, Tumlinson et al. (2017) likened the CGM to a galactic utility provider, acting as the galactic fuel tank, waste dump, and recycling center simultaneously.

The CGM is diffuse and therefore difficult to detect in emission, and has traditionally received less attention than the ISM and the intergalactic medium (IGM). However, interest in the CGM has undergone somewhat of a revolution in recent years. Galactic gas flows, which by necessity must pass through the CGM, appear to be at the heart of a myriad of unresolved and compelling issues, including the "missing baryon" problem (e.g., Keeney et al. 2017), the metal census (e.g., Peeples et al. 2014), the quenching of star formation in passive galaxies, and the perpetuation of star formation in star-forming galaxies (the so called red-blue dichotomy e.g., Bordoloi et al. 2011; Borthakur et al. 2016).
Advances in the modeling of galactic flows have prompted large strides forward in the observational capabilities to detect them, notably with the Cosmic Origins Spectrograph (Tumlinson et al. 2013; Werk et al. 2013) on the Hubble Space Telescope (HST). Evidence for accretion flows comes from line widths in $\mathrm{HI}$ and various other species, which reveal velocity dispersions in halo gas that show it to be bound (e.g., Tumlinson et al. 2013; Bordoloi et al. 2014; Ho et al. 2017). In the Milky Way, unmistakable evidence for accretion comes in the form of blueshifted high-velocity clouds (e.g., Sembach et al. 2004; Lehner \& Howk 2011). In other galaxies, the evidence has been more difficult to ascertain, presumably because individual flows contain insufficient mass or are too diffuse or both (Rubin et al. 2012). There are a handful of examples in which diffuse gas has been detected in emission around other galaxies (Cantalupo et al. 2014; Martin et al. 2014; Hennawi et al. 2015; Fumagalli et al. 2017) but for the most part the community has relied on absorption line spectroscopy (Rubin et al. 2012; Bouché et al. 2016; Wiseman et al. 2017).

On the other hand, observations of pristine gas in the IGM are rare (Fumagalli et al. 2011) and the metallicity of the gas in all phases of the CGM (Tumlinson et al. 2013; Peeples et al. 2014; Prochaska et al. 2017) is a sure indication that it has at some point passed through the ISM. This evokes a picture in which a significant amount of accreted material is recycled and blown out of the ISM and into galactic halos via feedback-triggered outflows. The evidence for outflows is large (Steidel et al. 2010; 
Rubin et al. 2014; Fox et al. 2015; Wiseman et al. 2017). What is less well known is how outflows interact with the CGM, and beyond, to transport processed matter and energy, and to what extent these processed materials are recycled in subsequent star formation.

The extensive progress of this field in recent years is examined comprehensively in a number of recent reviews (Putman et al. 2012; Fox and Davé 2017; Tumlinson et al. 2017). However, a complete picture of the CGM in the context of galaxy evolution is unachievable without broadening the discussion toward the extreme and evanescent phases of galaxy evolution such as nuclear starbursts, mergers, and active galaxies or quasars. The latter has been the subject of a series of nine papers known as the Quasars Probing Quasars (QPQ) project (Hennawi et al. 2006a; Hennawi \& Prochaska 2007; Prochaska \& Hennawi 2009; Hennawi \& Prochaska 2013; Prochaska et al. 2013b, 2013a, 2014; Lau et al. 2016, 2017, henceforth QPQ1-QPQ9). Just as the cosmic web or a galactic halo can be observed in absorption against the continuum emission of a distant quasar, so too can the CGM of a quasar host be observed when a background quasar is projected close to the line of sight of a foreground quasar. This is in fact the idea behind the QPQ project, which aims to elucidate the CGM in the massive $M \approx 10^{12.5} M_{\odot}$ halos that host quasars at $z \gtrsim 2$ (e.g., Eftekharzadeh et al. 2015).

A reoccurring conclusion throughout the QPQ series is that a quasar's ionizing continuum illuminates surrounding gas in an anisotropic fashion. Excess absorbers are found transverse to quasar sightlines (QPQ1) and the quasar-absorber clustering signal measured in transverse directions has been shown to overpredict the number of absorbers in line of sight directions by several times (QPQ2). This result holds to Mpc scales (QPQ6) and beyond (Font-Ribera et al. 2013, D. Sorini et al. 2018, in preparation). Furthermore, if this cool gas were to be illuminated by the quasar radiation field then it should emit Ly $\alpha$ photons, either from fluorescent recombinations, resonant scattering, or by Ly $\alpha$ cooling radiation. This has only rarely been found on large scales (QPQ4; Cantalupo et al. 2014; Martin et al. 2014; Hennawi et al. 2015). Other lines of inquiry have failed to detect the transverse proximity effect in background quasar spectra despite numerous attempts (Fernandez-Soto et al. 1995; Liske \& Williger 2001; Croft 2004; Schirber et al. 2004; Prochaska et al. 2013b).

Large covering fractions of $\mathrm{H}$ I extend to impact parameters of $R_{\perp} \lesssim 200 \mathrm{kpc}$ likely coinciding with the virial radii of halos (QPQ5; QPQ6; QPQ8; QPQ7). This gas represents up to a third of the total baryonic mass within the CGM, approaching the mass of baryons in the ISM (QPQ7). Beyond $R_{\perp} \gtrsim 500 \mathrm{kpc}$ the mass of cool gas remains substantial. If it is possible to generalize these findings to all coeval massive galaxies, then a significant amount of all optically thick gas is found within the extended regions around massive galaxies at $z \approx 2$ (QPQ6). Indeed, this phenomenon has no obvious affinity with coeval galaxy populations in other mass regimes (QPQ7) and must surely indicate a divergence of evolutionary processes.

Clues to the provenance of this cool halo gas can be explored via its metal content. Incidences of low-ion metal absorption systems follow closely with the results of $\mathrm{HI}$ above, i.e., the gas is enriched out to impact parameters of at least $1 \mathrm{Mpc}$ and shows the strongest signatures of metal enrichment at $R_{\perp} \lesssim$ 200 kpc (QPQ5; QPQ6; QPQ8; QPQ7). Stacked spectra reveal average profiles of both low and high ions with systematically larger equivalent widths than any other known galaxy population (QPQ7). Strong signatures of $\alpha$-elements implicate core-collapse supernovae as the progenitors of this gas and point to star formation histories similar to massive ellipticals, which are thought to be the modern-day descendants of quasars (QPQ8; QPQ9). This may link the enrichment of the CGM in quasar hosts at least in part to their own ISM but poses further questions regarding transport mechanisms.

Both quasar and star formation feedback are invoked as the transport mechanisms required to move gas out of the ISM and into the CGM. However, QPQ finds no obvious evidence for any single dominant process. While kinematics are extreme and in some cases suggestive of violent outflow (QPQ3; QPQ8), on the whole there is no need to appeal to anything beyond the gravitationally supported dynamics expected of massive halos (QPQ7; QPQ9). Simple arguments concerning timescales and energetics show that episodes of quasar activity are insufficient to place metals at $\sim \mathrm{Mpc}$ distances within a single duty cycle (QPQ3; QPQ7). Furthermore, cool gas is present, albeit in lower quantities, in the halos of quiescent galaxy populations, so ongoing quasar activity is not a prerequisite for its existence. On the other hand the average gas covering fraction may be correlated with quasar bolometric luminosity (Johnson et al. 2015), making it difficult to argue against the connection between cool halo gas and quasar activity. Far from ruling out quasar feedback as a transport mechanism completely, it is more appealing to invoke it as an intermittent agent operating on $\sim 100 \mathrm{kpc}$ scales.

Outflows supported by star formation feedback are found in a variety galaxy populations across time (Pettini et al. 1998; Martin 2005; Weiner et al. 2009; Rubin et al. 2014). Ongoing star formation however, does not appear to be precondition of a cool enriched CGM. Early type galaxies contain a large reservoir of cool enriched halo gas (Thom et al. 2012) and there is scant evidence to link the strength or prevalence of optically thick absorbers to star formation rate (QPQ7). Indeed $z \sim 2$ quasar hosts do not show evidence for increased star formation (Rosario et al. 2013; Stanley et al. 2017). Furthermore, the large impact parameters to which cool gas extends put it beyond the influence of any single star formation episode. The presence of heavy elements in the CGM serves as strong evidence that it has at some point passed through the ISM, but as with quasar feedback, this is likely to happen over integrated episodes limited to $\sim 100 \mathrm{kpc}$ scales.

The presence of heavy elements in the cool halo gas on Mpc scales means that it cannot be entirely explained by accretion of pristine gas from the cosmic web. Rather, it may arise from galactic winds driven by star formation in low-mass satellite galaxies that deposit their metals in cosmic filaments. Models of these processes have begun to be able to match the covering fractions of cool gas seen in observations (Faucher-Giguère et al. 2016), which alleviates the need for feedback processes in the host galaxy to act at these distances.

The QPQ series has contributed to a boom in the understanding of the CGM in the massive galaxies that host quasars at $z>2$. To facilitate this work the project has put together a database of over 2000 projected quasar pairs with transverse separations at foreground quasar redshifts ranging from between tens of physical kpc to a few Mpc. Equivalently, the database probes scales as small as a galactic disk, to unbound gas in the surrounding IGM, to everything in 
between. The QPQ collaboration here presents its extensive database of quasar pairs and their spectra. This submission adopts the $\Lambda \mathrm{CDM}$ cosmology of Planck Collaboration et al. (2016) and reports all magnitudes on the AB system. Section 2 recalls the basic principles behind the QPQ experiment, Section 3 discusses the selection of quasar pairs in spectroscopic redshift surveys, Section 4 discusses quasar pair selection in imaging data, Section 5 presents recent results from the latest QPQ quasar pair search using ATLAS, SDSS, and Wide-field Infrared Survey Explorer (WISE) imaging, Section 6 describes the content and architecture of the quasar pair catalog and accompanying spectral database, and Section 7 provides a summary of this paper and lays out some avenues for the future exploitation of the QPQ database.

\section{The Experiment}

To begin, it is useful to briefly review the basic principle behind the QPQ experiment. The upper panel of Figure 1 shows the projected quasar pair J1204+0221 in SDSS (Sloan Digital Sky Survey; York et al. 2000) imaging. The pair members are separated by an angular distance of $\theta \simeq 13$ ". 28 on the sky and are located at foreground and background redshifts of $z=2.44$ and $z=2.53$, respectively. From the adopted cosmology it follows that the sightline of the background quasar passes the foreground quasar at a transverse distance of $R_{\perp} \simeq 110$ pkpc. On their way to the observer, background quasar photons are absorbed and scattered by gas associated with the foreground quasar's halo and may ionize H I and other atomic species. The absorption features are seen as electronic transitions, primarily at far-ultraviolet (UV) rest-frame energies, in the spectrum of the background quasar at transition wavelengths corresponding to the redshift of the absorbing gas. This is shown in the lower panel, where Ly $\alpha$ absorption is clearly seen in the background spectrum at the redshift of the foreground quasar. This is accompanied by a series of labeled metal transitions.

A major goal of the QPQ project has been to search for projected quasar pairs in optical spectroscopic and photometric surveys. The blue cutoff in the optical $u$ band falls at $\sim 3500 \AA$, which corresponds to the wavelength of the redshifted Ly $\alpha$ transition at $z \sim 2$. This is the limit at which one can reasonably detect atomic hydrogen at optical wavelengths and in large, ground-based surveys such as the SDSS. QPQ pair searches have therefore been focused toward pairs with foreground members within this redshift regime. Inevitably, however, pairs outside of this redshift regime have found their way into the QPQ sample as false positives. These pairs are also included in the database, as are close physical binaries and gravitational lens candidates.

\section{Quasar Pair Selection from Spectroscopic Surveys}

A projected quasar pair is confirmed once spectra have been obtained that unambiguously reveal the spectral type of each pair member. In an age where spectroscopic redshift surveys have become a mainstay in extragalactic exploration, numbers of known quasar pairs have seen a corresponding spurt in growth.

Over the years, public spectroscopic survey projects have observed and cataloged over half a million quasars to redshifts
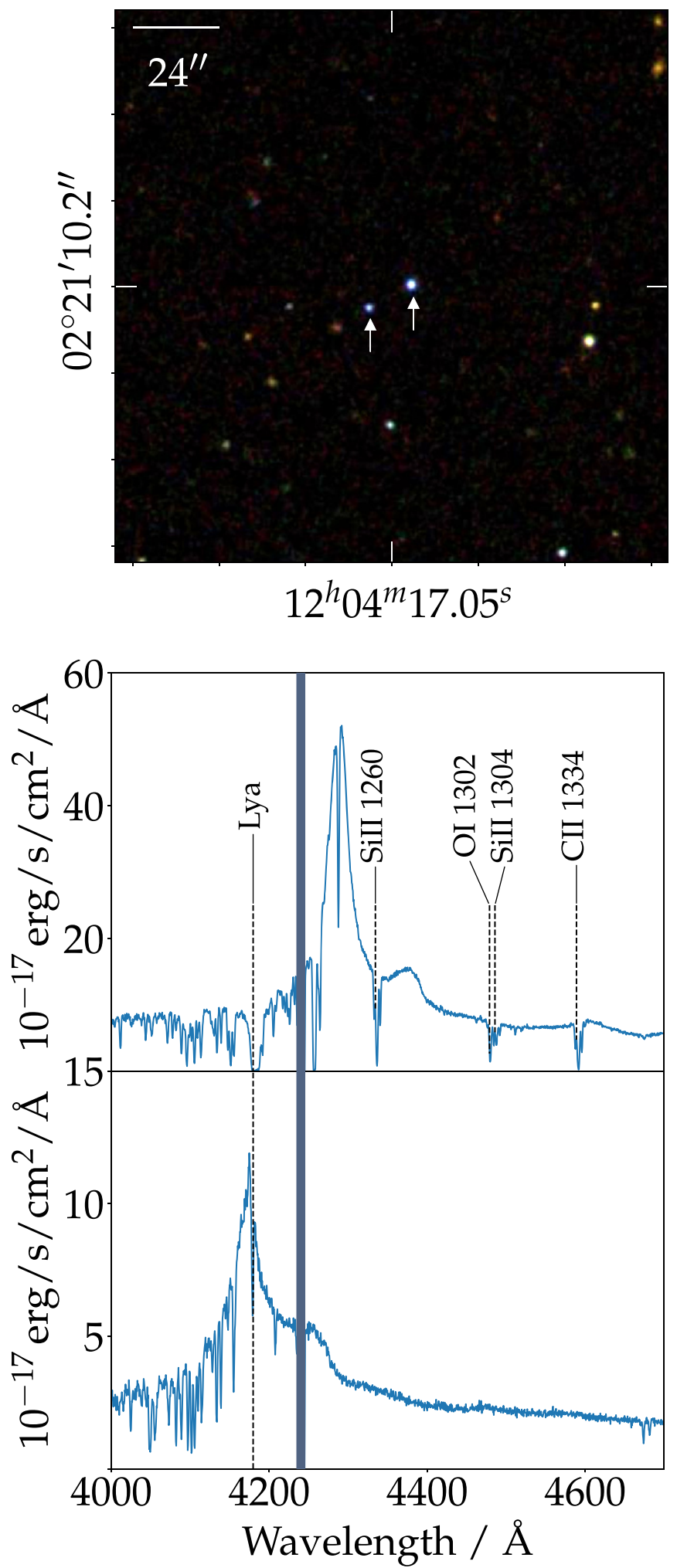

Figure 1. Illustration of the basic observation behind the QPQ experiment. The upper panel shows the projected quasar pair J1204+0221 at redshifts of $z=2.44$ and $z=2.53$. Photons from the background quasar pass through the halo of the foreground quasar and ionize $\mathrm{HI}$ and other atomic gas on their path. In the lower panel this process manifests in the form of absorption line transitions in the spectrum of the background quasar (upper) at transition wavelengths corresponding to the redshift of the foreground quasar (lower). The shaded areas mask regions of bad data. 
in excess of $z=7$. Unquestionably, the largest compendium of quasar spectroscopy has been provided by the SDSS. Using straightforward spatial matching, QPQ has relied heavily on the annual spectroscopic data releases of the SDSS to select quasar pairs at separations of $\gtrsim 1^{\prime}$. Due to fiber collisions, some pairs at closer separations require alternate techniques, which are discussed later in this section.

\subsection{The SDSS Legacy Surveys}

The original SDSS (York et al. 2000) ran between the years 2000-2008 and provides both imaging and spectroscopic coverage over $\sim 12,000 \mathrm{deg}^{2}$ of the sky. The two components of the survey, SDSS-I and SDSS-II, ran consecutively on a dedicated $2.5 \mathrm{~m}$ telescope located at the Apache Point Observatory, in New Mexico (Gunn et al. 2006). Collectively, these surveys are now known as the SDSS Legacy Survey (SDSS-LS).

SDSS-I ran during the first five years of operation and carried out multicolor $u$ (3551 ̊), $g$ (4686 $\AA$ ), $r$ (6166 $\AA$ ), $i(7480 \AA)$, and $z(8932 \AA)$ (Fukugita et al. 1996; Stoughton et al. 2002; Doi et al. 2010) imaging and targeted spectroscopic follow-up within the imaging footprint. SDSS-II extended the SDSS-I imaging footprint toward the Galactic plane.

The imaging and astrometric pipelines are described by Lupton et al. (2001) and Pier et al. (2003), respectively. Photometric calibration is tied to a standard star network (Hogg et al. 2001; Smith et al. 2002) and is refined to the $\sim 1$ percent level via a global re-calibration (Padmanabhan et al. 2008). The $95 \%$ completeness point source imaging depths are $u=22.0$, $g=22.2, r=22.2, i=21.3$, and $z=20.5$.

\subsection{The SDSS Baryon Oscillation Spectroscopic Survey (BOSS)}

The SDSS-LS was followed by SDSS-III, incorporating BOSS (Dawson et al. 2013), which was designed to measure the scale of baryon acoustic oscillations using quasars to trace the clustering of mass. SDSS-III extended the multicolor imaging of the SDSS-LS by a further $\sim 2500 \mathrm{deg}^{2}$ with the same telescope and camera between the years 2008-2009. All the SDSS imaging data were uniformly reduced with an improved sky subtraction and released under DR8 (Aihara et al. 2011), bringing the total imaging footprint to $14,555 \mathrm{deg}^{2}$.

The remainder of the survey (2009-2014) was devoted to spectroscopy. The SDSS-LS spectrographs were upgraded (Smee et al. 2013) with new higher-efficiency volume holographic gratings, fully depleted red CCDs with superior red response, blue CCDs with improved blue response and 1000 new fibers. Continuous wavelength coverage is provided between 3600 and 10,400 $\AA$ at resolutions between 1560 and 2270 in the blue channel and 1850-2650 in the red channel. Improved photometric selection algorithms were used to target 1.5 million luminous galaxies (Reid et al. 2016) and 150,000 new quasars in spectroscopy (Ross et al. 2012; Pâris et al. 2017).

\subsection{The 2dF QSO Redshift Survey}

Running in partial overlap with the SDSS surveys was the 2 degree Field (2dF) QSO Redshift Survey (2QZ; Croom et al. 2004). The QPQ search for quasar pairs in $2 \mathrm{QZ}$ was a similar search for spatially coincident catalog positions.
2QZ provides a homogeneous quasar catalog flux-limited to $16.00<b_{J}<20.85$. Candidates were color- selected via multiwavelength $u, b_{J}, \quad r$ photometry from automated plate measurement of UK Schmidt Telescope (UKST) photographic plates. These candidates were then observed by the $2 \mathrm{dF}$ instrument, a multi-object spectrograph at the Anglo-Australian Telescope (AAT). The 2QZ catalog comprises of 23,338 quasars spanning a redshift range of $0.3 \lesssim z \lesssim 3.0$. The footprint covers a total area of $721.6 \mathrm{deg}^{2}$ and is arranged over two contiguous strips of $75^{\circ} \times 5^{\circ}$ in area across the southern Galactic cap, centered on $\delta=-30^{\circ}$ and the northern Galactic cap centered on $\delta=0^{\circ}$.

\section{Quasar Pair Selection in Imaging Surveys}

The 2QZ and SDSS surveys have made searching for quasar pairs a trivial endeavor, but with the caveat that this method is inherently biased against close pairs due to the finite distance at which pairs of spectroscopic fibers can be placed from one another.

Approximately $30 \%$ of the SDSS-LS and BOSS are covered spectroscopically in more than one epoch. These regions do not suffer from the effects of fiber collisions and close quasar pairs in these footprints can be drawn directly from the spectroscopic catalogs. Additionally, the 2QZ NGC region shares approximately half of its footprint with SDSS-LS, allowing 2QZ quasars to be assigned SDSS quasar pairs.

For the most part, however, fiber collisions prevent objects with separations of $<55^{\prime \prime},<62^{\prime \prime}$, and $<30^{\prime \prime}$ from being observed simultaneously in SDSS-LS, BOSS, and 2QZ, respectively (Lewis et al. 2002; Blanton et al. 2003; Dawson et al. 2013). Therefore, in most cases, close quasar pairs are selected by spatially matching to photometrically targeted objects with quasar-like colors in close proximity to spectroscopically cataloged quasars. Photometric candidates are later followed up spectroscopically to confirm the pair.

QPQ has used SDSS imaging to select photometric quasar pair members in this way via three distinct methods. Hennawi et al. (2006b) described a means to select pairs at similar redshifts via a $\chi^{2}$ selection statistic, which utilizes the fact that the rest-frame UV-to-optical spectral energy distributions of quasars follow a remarkably tight color redshift relation. If one neglects the relatively small intrinsic scatter of the population about this relation, then the fluxes of a pair of quasars at the same redshift should be related by a single proportionality constant across all bands. Variation in this proportionality can then be approximately attributed to observational error. One can find the constant of proportionality that minimizes the sum of the differences in flux between pairs across all bands. In this way it is possible to efficiently select close pairs of quasars at similar redshifts.

To select close quasar pairs at differing redshifts, Hennawi et al. (2006b; see also Myers et al. 2007, 2008; Eftekharzadeh et al. 2017) made use of the various photometric catalogs collated by Richards et al. (2004, 2009a, 2009b, 2015). Richards et. al. used kernel density estimation to model the probability density functions of stars and quasars in the 4D SDSS color space. The likelihood that a given photometric object originates from either of these two distributions is combined with prior knowledge of quasar and stellar number densities using Bayes' theorem. The result is a probabilistic classification of an object as either a star or a quasar. QPQ mined these catalogs for both photometric-photometric and photometric-spectroscopic pairs 
with large quasar probabilities. Candidates were then followed up spectroscopically.

Bovy et al. (2011) compiled a targeting catalog of photometric quasar candidates for BOSS using a nonparametric Bayesian classifier that represents an advance on the Richards et al. technique by approximating the underlying density distributions of stars and galaxies in flux space via an extreme-deconvolution. The classification code, XDQSO, was extended to provide probabilistic selection over arbitrary redshift intervals, as well to incorporate UV and near- infrared (IR) information (XDQSOZ; Bovy et al. 2012). Further details on the continuing effort to discover both photometric-spectroscopic and photometric-photometric quasar pairs are found in Hennawi (2004) and Hennawi et al. (2006b, 2010).

\section{New Pairs from SDSS, ATLAS and WISE}

The most recent QPQ search for photometric-photometric pairs was conducted in optical imaging from the SDSS and VST ATLAS (Shanks et al. 2015) surveys, combined with midIR data from the Wide-field Infrared Survey Explorer (WISE; Wright et al. 2010). Unlike the searches described in previous sections, this search has not been discussed in any previous publication. It is described here in detail for the first time.

\subsection{The VST ATLAS Survey}

ATLAS is an optical $u, g, r, i, z$ survey carried out by OmegaCam (Kuijken 2011) on the European Southern Observatory's (ESO) $2.61 \mathrm{~m}$ VLT Survey Telescope (VST; Schipani et al. 2012) at the Cerro Paranal Observatory in Chile. ATLAS has completed its final $4711 \mathrm{deg}^{2}$ footprint in all filters over two contiguous regions covering the northern and southern Galactic caps during 6 years of observations. The premise of the ATLAS survey is to provide imaging in the southern hemisphere with equivalent depth and better image quality than SDSS.

The ATLAS data is reduced by end-to-end astrometric and photometric pipelines run by the Cambridge Astronomical Survey Unit $^{10}$ (CASU) and archived by the Wide Field Astronomy Unit in the OmegaCam Science Archive ${ }^{11}$ (OSA).

\subsection{The Wide-field Infrared Survey Explorer}

WISE was launched in 2009 December, and from 2010 February to August, it surveyed the entire sky in four mid-IR bands, W1 $(3.4 \mu \mathrm{m}), W 2(4.6 \mu \mathrm{m}), W 3(12 \mu \mathrm{m})$, and $W 4$ $(22 \mu \mathrm{m})$. This initial survey release, termed the "AllSky" release, represents a significant step forward in exploration of the mid-IR sky at these wavelengths. The $5 \sigma$ AB point source sensitivity limits are deeper than 19.1, 18.8, 16.4, and 14.5 mag in W1-W4, respectively, providing over 100 times the sensitivity of the $12 \mu \mathrm{m}$ band of the InfraRed Astronomical Satellite (Neugebauer et al. 1984).

In 2010 September the cryogen cooling the W3 and W4 instruments depleted, ending the full four-band mission. The W1 and W2 band missions continued through 2011 February. encompassing both the AllWISE and Near Earth Object WISE (NEOWISE; Mainzer et al. 2011) data releases. After a period of hibernation this was followed by the NEOWISE

\footnotetext{
${ }^{10}$ http://casu.ast.cam.ac.uk/

11 http://osa.roe.ac.uk/
}

Reactivation mission (NEOWISER; Mainzer et al. 2014) in 2013 October, which is still in operation at the time of writing (2018 March).

\subsection{Data Preparation and Candidate Selection}

SDSS and ATLAS differ widely from WISE in depth, resolution, and wavelength, and as a result there are significant benefits to forced photometry in WISE images at optical positions compared to traditional positional catalog-matching. The angular resolution of the WISE imaging is diffractionlimited and at long mid-IR wavelengths this translates into a resolving power of several arcseconds. Conversely, the angular resolutions of the optical imaging catalogs are seeing-limited, translating to roughly 1"! 0 at the median. Forced photometry ensures that the optical and WISE measurements are linked to a consistent set of sources, whereas catalog-matching would inevitably lead to a tail of wide-separation erroneous positional matches.

Furthermore, the bulk of SDSS and ATLAS quasar candidates lie toward the limiting depths of the respective catalogs. Many of these sources are undetected at the shallower limiting depths of the AllWISE public release catalogs. Measurements of these faint or even undetected sources in WISE are just as scientifically valuable as a significant detection, particularly for the sophisticated statistical selection techniques described in Section 4.

The full-depth coadded WISE images are available as part of the AllWISE data release. These full-depth images are convolved by the point-spread function (PSF) during the coaddition process. This step is included to improve the detection of isolated point sources, but it is inappropriate for other applications such as forced photometry, since the blurring of the PSF decreases the available signal-to-noise. Lang (2014) provided an independent reduction of the AllWISE data products, which consists of full-depth coadds at the full instrument resolution. These data products, termed the "unWISE" coadds, were used to assemble a catalog of midIR photometry forced at the sites of over 400 million SDSS sources (Lang et al. 2016). Closely following this release, the XDQSOZ selection code was updated to incorporate the unWISE imaging and a catalog of over 5 million photometric quasar candidates was generated over the SDSS footprint (DiPompeo et al. 2015). This candidate list served as the starting point for the photometric pair search in SDSS and WISE.

More recently, Meisner et al. (2017) have undertaken further reprocessing of AllWISE, incorporating three years of NEOWISER imaging into the unWISE framework. The coadded data products provide significant gains in depth over the original unWISE release, and in addition, time-dependent artifacts such as moon contamination are largely eliminated because of the inclusion of multiple epochs.

Band-merged ATLAS source catalogs provided by CASU were force-photometered at the $W 1$ and $W 2$ bandpasses in the unWISE coadds of Meisner et al. (2017). The forced photometry was performed by the TRACTOR (D. Lang et al. 2018 , in preparation). The TRACTOR is an innovative code for inference-modeling of astronomical sources. Tractor optimizes the likelihood for the source properties given a set of imaging data and an informative noise model. The details of the TRACTOR implementation running in forced photometry mode 
on the unWISE coadds were discussed by Lang et al. (2016). The QPQ TRACTOR run follows this closely.

QPQ used the updated XDQSOZ selection code to construct a catalog of quasar candidates from ATLAS and forced unWISE imaging over a fraction of the ATLAS footprint. This catalog, along with the SDSS-unWISE catalog described above, were then mined for close pairs of objects with $>90 \%$ quasar probabilities. Candidates were selected to $r<22$ but emphasis was typically placed on selecting pairs with $r<21$ and with foreground members at $z>3$. All pairs within $1^{\prime}$ were considered for follow-up, but priority was given to pairs within $<30^{\prime \prime}$.

The benefits of combining WISE mid-IR data into the established ugriz optical quasar selection method have been explored since the WISE AllSky data release in 2012 March (e.g., Wu et al. 2012). Among the first projects to explore this practically were the SDSS-IV Extended Baryon Oscillation Spectroscopic Survey (eBOSS; Myers et al. 2015) and the 2dF Quasar Dark Energy Survey pilot (2QDESp; Chehade et al. 2016), a spectroscopic survey of quasar candidates selected in ATLAS and WISE imaging. These projects showed the utility of the infrared excess in quasar SEDs for achieving large separations between the quasar and stellar loci. The utility of the WISE mid-IR imaging can be seen in Figure 2, where the quasar pair database is plotted alongside stars for four different color-color combinations, including the SDSS and WISE WI passbands. Only where WISE data is incorporated, as in the lower right panel, is there a significant distinction between the stellar and quasar loci.

\subsection{Spectroscopic Follow-up}

Candidates at decl. $>-20^{\circ}$ (all those presented here) were observed on the Intermediate dispersion Spectrograph and Imaging System (ISIS; Jorden 1990), mounted at the Cassegrain focus of the $4.2 \mathrm{~m}$ William Herschel Telescope (WHT). The observing run was conducted on 22 nights between 2015 December and 2016 March as part of program 2015/P1 and 2016/P6. Approximately three nights were lost due to poor weather. Usable nights were for the most part clear but not always photometric.

The instrument was configured with R300B and R316R gratings centered at 4230 and $6400 \AA$ in the blue and red arms, respectively, with the D5300 dichroic filter in place over both channels. A $1^{\prime \prime}$ slit width, combined with a factor 2 binning in the dispersion direction at readout time, resulted in continuous wavelength coverage over 3000-7630 $\AA$, with average resolutions of $R \sim 4.1 \AA$ and $R \sim 3.8 \AA$ in the blue and red channels, respectively.

The data were reduced using the Low-Redux pipeline ${ }^{12}$ extended to work with the ISIS detectors. This pipeline performs basic calibrations (bias, flat-fielding, and wavelength calibration), and extracts flux-calibrated 1D spectra, which are then coadded across multiple exposures. All spectra were visually inspected to separate stars from quasars, and to measure the quasar redshifts by superposing a quasar template to the data.

In total, 69 photometrically selected pairs in ATLAS were observed on WHT, resulting in 15 new projected quasar pairs. The remaining observing time was devoted to observations of

\footnotetext{
12 http://www.ucolick.org/ xavier/LowRedux/
}

candidates selected from SDSS, yielding the discovery of 39 new pairs.

The foreground quasars are distributed between $z \approx 0.8-3.6$ and their halos are probed by their background counterparts over physical scales of $R_{\perp} \approx 0.1-0.5 \mathrm{Mpc}$. The coordinates, redshifts, on-sky, and physical separations are given for the new quasar pairs in Table 1. The star symbols in Figure 2 show the positions of the new projected quasars in ugriz W1 colorcolor space and are color-coded according to their redshifts.

\section{The Quasar Pair Database}

The database of quasar pairs comprises a catalog and a spectral library, both housed within a single HDF5 SPECDB file. SPECDB is a software package ${ }^{13}$ for generating and interfacing with databases of astronomical spectra, written and maintained by JXP. The following sections focus on the database content, source, and data characteristics and the database architecture. Full documentation of the SPECDB package, including download instructions for the spectral database, are supplied by Read the Docs. ${ }^{14}$ We further note that the IGMSPEC database, also under SPECDB, provides approximately 500,000 quasar spectra from public and private data sets (Prochaska 2017). In keeping with its goals, which include maintaining a highly complete database of quasar spectra in a consistent format, the quasar pair spectra will also be ingested into IGMSPEC; however, some of the catalog content will only be available via the QPQ pair catalog presented here.

\subsection{The Catalog}

The quasar pair catalog comprises a simple table containing a single record for each unique source. Distinct catalog records are uniquely identified via a primary key. The catalog also contains celestial coordinates, redshifts, and references to all three, as well as a redshift uncertainty column, which remains empty, and UV and mid-IR photometry for all pairs within $5^{\prime}$ of separation. The celestial coordinates come largely from PanStarrs (Chambers et al. 2016), because except for a small fraction of objects that fall outside of the footprint, PanStarrs covers the entire catalog with sub-arcsecond accuracy.

The catalog redshifts are estimated via a wide range of distinct methodologies from a variety of sources including cross-correlation (Hewett \& Wild 2010), principle component analysis (Pâris et al. 2012), spectral line fitting (QPQ6), and visual inspection (this submission). The most significant difficulty in estimating quasar redshifts is accounting for the natural variance in the emission-line properties of the quasar population both as a function of redshift and luminosity. This variation is large and ill-understood and some redshift estimators are better than others in accounting for it. Consolidating redshift uncertainties with differing systematics into a single table would be inconsistent and misleading. Instead, redshift uncertainties have been estimated at $\delta \simeq 1000 \mathrm{~km} \mathrm{~s}^{-1}$, which is conservative. ${ }^{15}$ The redshift uncertainty column is empty but remains in the catalog to

\footnotetext{
13 https://github.com/specdb/specdb

$14 \mathrm{http}: / /$ specdb.readthedocs.io/en/latest/

15 Note also that Shen et al. (2016) give good general guidelines for the uncertainty associated with particular lines, which may be used to estimate the uncertainty at a given redshift, simply by considering which lines are redshifted into the optical window.
} 

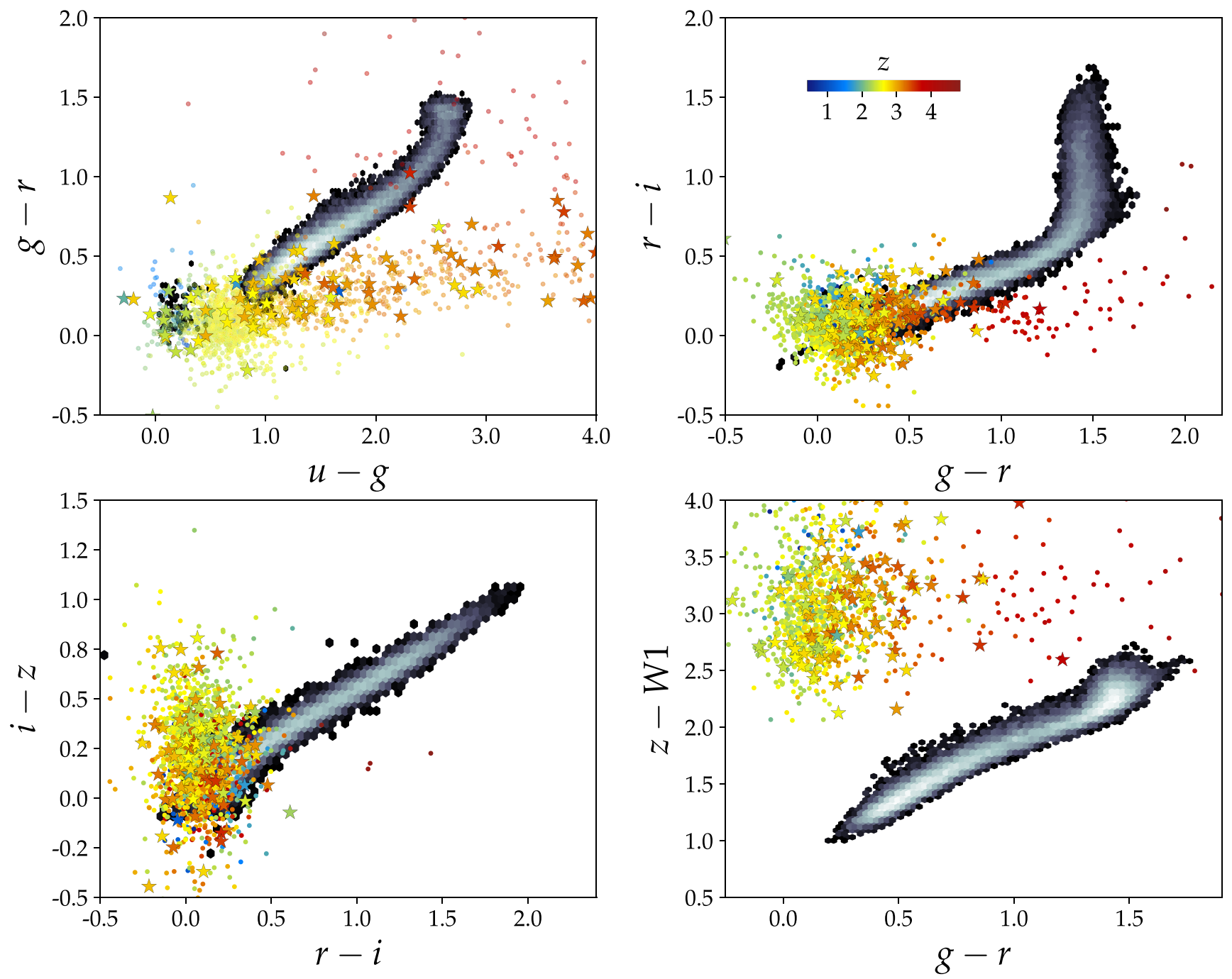

Figure 2. Color-color plots showing the stellar locus in grayscale and the quasar pair database color-coded according to redshift. The benefit of introducing the WISE W1 band to SDSS ugriz color combinations is seen in the bottom right hand panel, where quasars and stars occupy distinct regions of color-color space. The starshaped symbols show quasar pairs discovered during the recent QPQ search in ATLAS, SDSS, and WISE imaging. The circles show the remainder of the QPQ pair database. No attempt has been made here to place the ATLAS photometry onto the SDSS system; at the scales presented here the differences are completely negligible.

facilitate future attempts to measure redshifts more consistently (see Section 7).

Columns of mid-IR and UV photometry are provided via measurements made at the positions of catalog objects in the unWISE coadds (Section 5) and the Galaxy Evolution Explorer (GALEX; Morrissey et al. 2007) imaging products. Forced photometry from these two surveys is included for the reasons discussed in Section 5.

GALEX undertook wide-field surveys in both imaging and low-resolution grism spectroscopy from 2003 May until 2012 February. It delivered the first broadband imaging surveys in the far-UV and near-UV at central wavelengths of $1528 \AA$ and $2310 \AA$, respectively.

Flux-calibrated, background-subtracted intensity maps, as well as the sky background and threshold weight maps are served by the Barbara A. Mikulski Archive for Space Telescopes (MAST). The GALEX photometry pipeline (Morrissey et al. 2007) passes these products through SEXTRACTOR (Bertin \& Arnouts 1996) to obtain calibrated catalogs. MAST also serves the SEXTRACTOR configuration and parameter files necessary for computing photometry on each individual image. In principle then, one should be able to reconstruct any GALEX catalog in the official release. The slight complication with forced photometry is the need to bypass the source detection stage and simply place apertures down at predefined pixel positions. SEXTRACTOR does not have this facility and therefore forced photometry was performed by constructing mock images with mock sources at the position of the object of interest. SEXTRACTOR was then run in dual image mode, which allows the mock image to be used for the purposes of source detection and the real image to be used for the source extraction, thereby extracting the real source from the real image at the position of the mock source in the mock image. Beyond this modification the procedure follows that of the actual GALEX photometry pipeline. Photometry is provided for a $6^{\prime \prime}$ radius aperture, which is a reasonable compromise between minimizing background noise contributions and measuring photometry toward the field edges where the PSF becomes degraded.

The results of the unWISE and GALEX forced photometry are verified in Figures 3 and 4, respectively, where members of the quasar pair catalog that are detected in the officially 
Table 1

Table of New Quasar Pairs Discovered in Combined ATLAS, SDSS, and WISE Imaging

\begin{tabular}{|c|c|c|c|c|c|c|}
\hline $\mathrm{QSO}_{\mathrm{fg}}$ & $\mathrm{QSO}_{\mathrm{bg}}$ & $z_{\mathrm{fg}}$ & $z_{\mathrm{bg}}$ & $\theta^{\prime}$ & $R_{\perp}(\mathrm{pkpc})$ & Survey \\
\hline J003308.63-083222.19 & J003307.31-083241.55 & 3.038 & 3.043 & 0.459 & 216.433 & SDSS \\
\hline J012902.78+191824.46 & J012901.92+191847.18 & 2.680 & 2.691 & 0.430 & 209.778 & SDSS \\
\hline $\mathrm{J} 015415.22+032455.84$ & J015416.43+032457.86 & 2.660 & 3.219 & 0.304 & 148.640 & SDSS \\
\hline J022845.72-124643.92 & J022848.07-124706.78 & 1.733 & 2.032 & 0.688 & 358.303 & ATLAS \\
\hline J023229.05-100123.48 & J023231.25-100102.92 & 2.063 & 2.386 & 0.641 & 328.856 & ATLAS \\
\hline J031855.31-103040.30 & J031853.87-102945.32 & 2.226 & 2.417 & 0.982 & 498.485 & ATLAS \\
\hline J032926.40-134732.22 & J032926.04-134831.51 & 2.073 & 2.372 & 0.992 & 508.701 & ATLAS \\
\hline J033347.40-133928.44 & J033345.40-133938.41 & 2.230 & 2.679 & 0.513 & 260.495 & ATLAS \\
\hline J034952.34-110620.59 & J034955.77-110642.91 & 2.449 & 2.824 & 0.920 & 458.622 & ATLAS \\
\hline J090551.96+253003.35 & J090551.25+253026.09 & 3.325 & 3.300 & 0.411 & 188.462 & SDSS \\
\hline J090828.30+080313.18 & J090826.82+080320.34 & 2.390 & 3.168 & 0.385 & 193.025 & SDSS \\
\hline J091800.77+153621.46 & $\mathrm{J} 091800.70+153631.31$ & 2.980 & 2.958 & 0.165 & 78.282 & SDSS \\
\hline J093240.91+400905.65 & J093243.02+400913.95 & 2.962 & 3.130 & 0.426 & 202.556 & SDSS \\
\hline J093836.78+100905.34 & J093837.81+100922.00 & 2.504 & 2.818 & 0.376 & 186.529 & SDSS \\
\hline $\mathrm{J} 095503.57+614242.66$ & J095503.14+614247.33 & 2.739 & 2.725 & 0.093 & 45.173 & SDSS \\
\hline J095549.38+153838.11 & J095549.80+153837.00 & 0.830 & 2.900 & 0.103 & 48.214 & SDSS \\
\hline J095629.72+243441.34 & J095627.88+243436.98 & 2.979 & 2.914 & 0.425 & 201.423 & SDSS \\
\hline $\mathrm{J} 100205.70+462411.82$ & $\mathrm{~J} 100202.89+462407.25$ & 3.138 & 2.760 & 0.490 & 228.948 & SDSS \\
\hline $\mathrm{J} 100253.37+341924.03$ & $\mathrm{~J} 100254.22+341928.47$ & 2.418 & 2.506 & 0.190 & 95.194 & SDSS \\
\hline J100903.16-142104.27 & J100859.11-142114.19 & 2.033 & 2.068 & 0.995 & 511.335 & ATLAS \\
\hline J101853.24-160727.80 & J101853.10-160808.04 & 2.331 & 2.953 & 0.672 & 338.026 & ATLAS \\
\hline $\mathrm{J} 102947.32+120817.11$ & $\mathrm{~J} 102945.77+120824.53$ & 2.820 & 3.392 & 0.399 & 192.024 & SDSS \\
\hline $\mathrm{J} 103109.37+375749.68$ & $\mathrm{~J} 103108.25+375801.19$ & 2.752 & 2.589 & 0.292 & 141.842 & SDSS \\
\hline $\mathrm{J} 103716.68+430915.57$ & $\mathrm{~J} 103716.86+430944.83$ & 2.676 & 3.286 & 0.489 & 238.758 & SDSS \\
\hline $\mathrm{J} 104314.33+143434.81$ & $\mathrm{~J} 104313.69+143435.73$ & 2.980 & 3.361 & 0.156 & 73.812 & SDSS \\
\hline $\mathrm{J} 104339.12+010531.29$ & $\mathrm{~J} 104338.28+010507.77$ & 3.240 & 3.001 & 0.445 & 205.465 & SDSS \\
\hline J105202.95-103803.70 & J105203.23-103815.09 & 2.104 & 2.194 & 0.202 & 103.336 & ATLAS \\
\hline J105338.15-081623.66 & J105336.09-081620.94 & 2.192 & 2.294 & 0.512 & 260.282 & ATLAS \\
\hline J105354.90-100941.44 & J105354.48-100931.71 & 3.232 & 3.248 & 0.192 & 88.924 & ATLAS \\
\hline $\mathrm{J} 110402.08+132154.46$ & $\mathrm{~J} 110401.42+132134.70$ & 2.869 & 2.576 & 0.366 & 175.702 & SDSS \\
\hline J110124.79-105645.12 & J110126.03-105642.26 & 2.579 & 2.688 & 0.308 & 151.832 & ATLAS \\
\hline $\mathrm{J} 111820.36+044120.22$ & $\mathrm{~J} 111820.46+044125.26$ & 3.120 & 3.454 & 0.088 & 40.981 & SDSS \\
\hline J112032.04-095203.21 & J112032.65-095138.28 & 2.180 & 3.627 & 0.442 & 224.954 & ATLAS \\
\hline $\mathrm{J} 112239.32+450618.54$ & $\mathrm{~J} 112236.72+450628.12$ & 3.590 & 3.044 & 0.486 & 216.459 & SDSS \\
\hline $\mathrm{J} 112355.97-125040.73$ & J112359.53-125056.76 & 2.965 & 3.428 & 0.908 & 431.314 & ATLAS \\
\hline $\mathrm{J} 112516.06+284057.59$ & $\mathrm{~J} 112516.26+284122.74$ & 2.845 & 2.834 & 0.421 & 202.590 & SDSS \\
\hline J112839.64-144842.36 & J112843.30-144837.44 & 1.920 & 2.200 & 0.888 & 459.457 & ATLAS \\
\hline $\mathrm{J} 112913.52+662039.13$ & $\mathrm{~J} 112915.28+662101.63$ & 2.807 & 2.803 & 0.414 & 199.966 & SDSS \\
\hline $\mathrm{J} 113820.28+203336.93$ & $\mathrm{~J} 113820.42+203333.18$ & 2.687 & 2.679 & 0.071 & 34.437 & SDSS \\
\hline $\mathrm{J} 114443.59+102143.48$ & $\mathrm{~J} 114442.32+102125.21$ & 1.503 & 2.833 & 0.436 & 227.342 & SDSS \\
\hline $\mathrm{J} 115037.52+422421.01$ & $\mathrm{~J} 115035.53+422409.90$ & 2.883 & 3.126 & 0.411 & 197.017 & SDSS \\
\hline $\mathrm{J} 115222.15+271543.29$ & $\mathrm{~J} 115221.84+271540.80$ & 3.102 & 3.083 & 0.080 & 37.686 & SDSS \\
\hline $\mathrm{J} 120032.34+491951.99$ & $\mathrm{~J} 120034.26+492015.22$ & 2.629 & 3.254 & 0.498 & 244.193 & SDSS \\
\hline $\mathrm{J} 121642.25+292537.97$ & $\mathrm{~J} 121641.77+292529.34$ & 2.532 & 2.519 & 0.178 & 87.996 & SDSS \\
\hline $\mathrm{J} 122900.87+422243.23$ & $\mathrm{~J} 122859.36+422229.73$ & 3.842 & 3.459 & 0.358 & 155.535 & SDSS \\
\hline $\mathrm{J} 123055.78+184746.79$ & $\mathrm{~J} 123056.94+184736.83$ & 3.169 & 3.089 & 0.321 & 149.312 & SDSS \\
\hline $\mathrm{J} 132728.77+271311.96$ & $\mathrm{~J} 132729.83+271324.94$ & 3.085 & 2.658 & 0.320 & 150.152 & SDSS \\
\hline $\mathrm{J} 134221.26+215041.97$ & $\mathrm{~J} 134219.85+215051.20$ & 3.062 & 2.506 & 0.362 & 170.098 & SDSS \\
\hline $\mathrm{J} 135456.96+494143.74$ & $\mathrm{~J} 135456.76+494154.08$ & 3.126 & 2.928 & 0.175 & 81.962 & SDSS \\
\hline $\mathrm{J} 141457.24+242039.67$ & $\mathrm{~J} 141457.12+242106.23$ & 3.576 & 3.515 & 0.444 & 197.922 & SDSS \\
\hline $\mathrm{J} 143622.50+424127.13$ & $\mathrm{~J} 143622.01+424132.22$ & 3.000 & 3.050 & 0.124 & 58.564 & SDSS \\
\hline $\mathrm{J} 144225.30+625600.96$ & J144223.04+625625.99 & 3.271 & 3.271 & 0.490 & 225.685 & SDSS \\
\hline $\mathrm{J} 162413.70+183330.72$ & $\mathrm{~J} 162412.59+183348.25$ & 2.763 & 3.263 & 0.393 & 190.480 & SDSS \\
\hline $\mathrm{J} 214858.11-074033.28$ & $\mathrm{~J} 214858.06-074034.98$ & 2.660 & 2.660 & 0.031 & 15.128 & SDSS \\
\hline
\end{tabular}

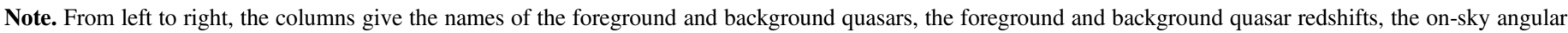

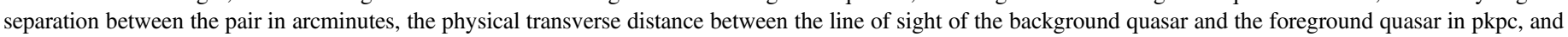
the survey in which the pair was discovered.

released AllWISE or GALEX catalogs are plotted against the forced photometry. In Figure 3 the left panels show the comparison of AllWISE and unWISE photometry and the orange lines show a one-to-one relationship. The plot axes extend to the average $3 \sigma$ depth of the official release in each band and the dashed lines show the average $5 \sigma$ depth in each band. The right panels compare magnitude errors. The benefits of using the unWISE products over the official AllWISE release are clear. Significant gains in signal-to-noise are achieved in all bands and particularly in $W 1$ and $W 2$ where 

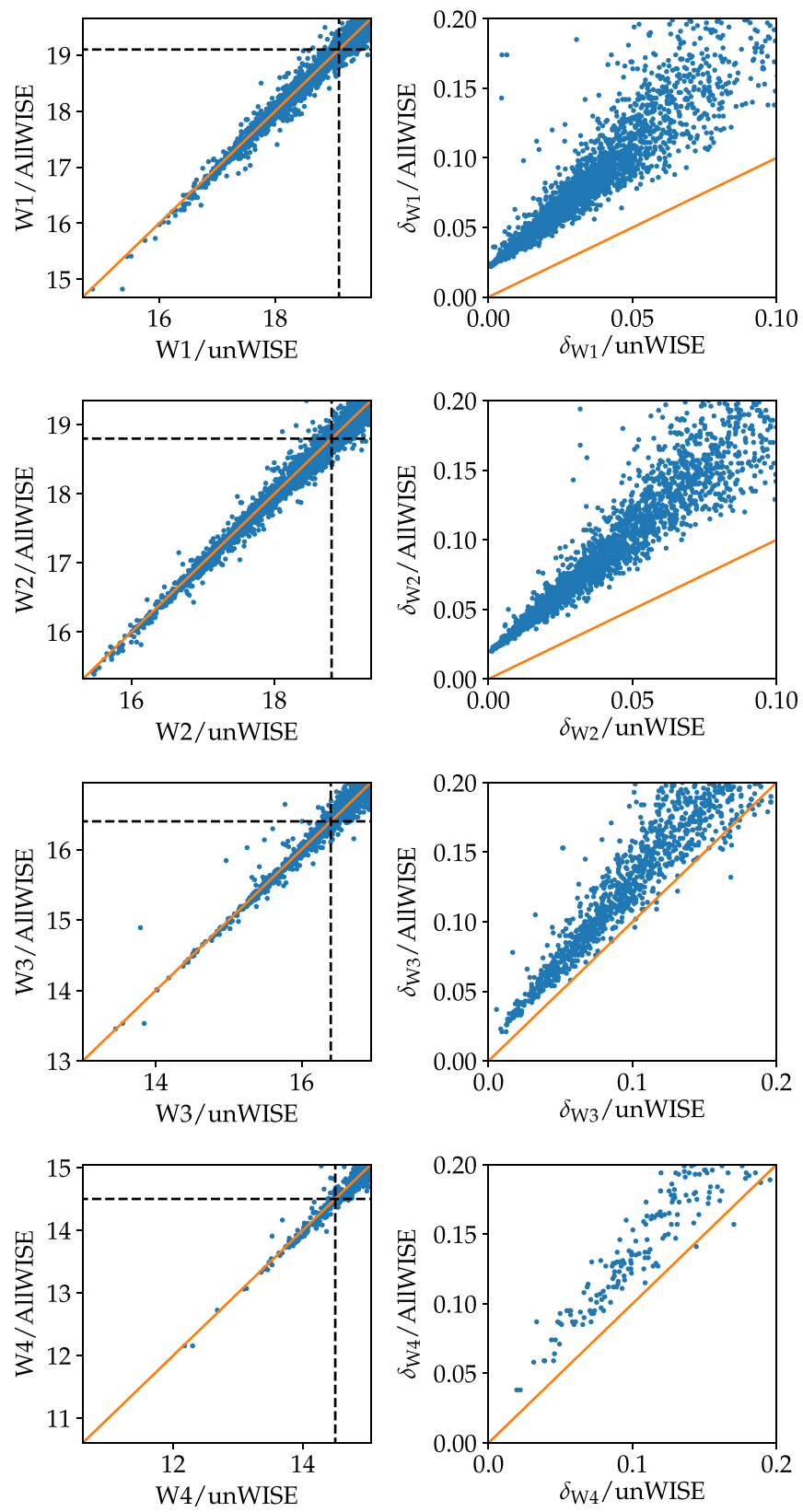

Figure 3. Comparison of the AllWISE photometry of detected quasar pairs with the forced photometry of the unWISE images. The left panels show the magnitude measurements from the AllWISE release vs. the forced measurements. The orange line shows a one-to-one relationship and the dashed lines are placed at the average $5 \sigma$ AllWISE limiting magnitudes. The right panels compare the magnitude errors. The gains in unWISE over the AllWISE data set are obviously apparent, especially in the $W 1$ and $W 2$ bands where the addition of NEOWISE and NEOWISER data has approximately doubled the signal-tonoise.

the additions of the NEOWISE observations have almost doubled the signal-to-noise over the AllWISE release.

Figure 4 shows a similar comparison between the officially released and forced GALEX photometry. The forced photometry in both cases, unWISE and GALEX, is in good agreement with their respective official releases. Forced photometry of quasar pairs in optical or near-IR surveys is omitted from the catalog since straightforward catalog-matching is both efficient and accurate at the fine spatial resolutions offered by modern surveys in these wavelength regimes.
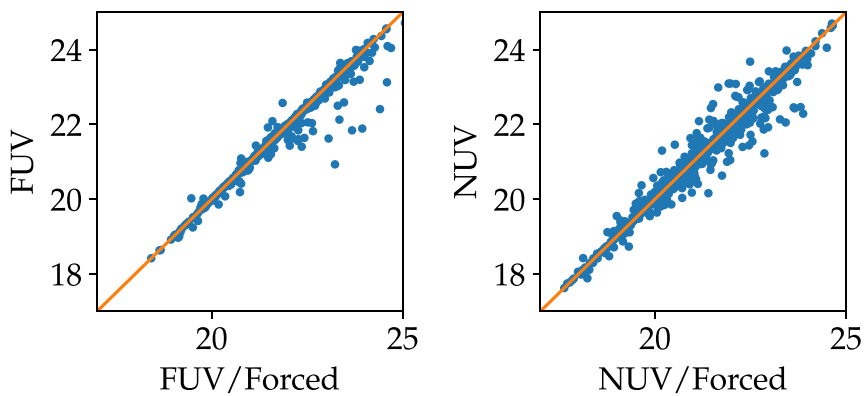

Figure 4. Comparison of officially released GALEX photometry for detected quasars vs. forced photometry. The left panel shows FUV photometry and the right panel shows NUV photometry. The orange line shows a one-to-one relationship and the two sets of measurements are in good agreement, which serves to verify the forced photometry method.

A full description of the catalog is given in Table 3. Figure 5 shows the sky coverage of the catalog with points plotted at the locations of all foreground quasars and color-coded according to redshift. The filled regions correspond to the imaging footprints of the surveys that bound the QPQ quasar searches, namely SDSS-LS, BOSS, ATLAS, and 2QZ. The background map shows the Milky Way polarized dust emission from the Planck commander component separation (Planck Collaboration et al. 2015).

The distribution of foreground quasar redshifts is shown in the right panel of the joint-plot in Figure 6. The distribution peaks at $z \sim 2.5$, corresponding to the peak in cosmic quasar activity. From the redshifts of foreground and background pair members and the known angular separations between them, one can compute the proper transverse separation of a foreground quasar to the sightline of the background quasar. The distribution of proper transverse separations is shown in the upper panel of Figure 6 and the joint distribution with redshift is shown in the main panel of the same figure. Physical binaries are omitted in each of these plots by cutting the sample to pairs with velocity differences of $>3000 \mathrm{~km} \mathrm{~s}^{-1}$. This plot serves to illustrate the range of environments probed by the background quasar sightlines. Distances of tens of pkpc, corresponding to the outer regions of galactic discs to hundreds of pkpc, probing the CGM, to a few pMpc corresponding to scales in the cosmic web, are all probed by the background sources.

\subsection{The Spectroscopic Library}

The spectroscopic library houses the spectra of quasar pairs in the catalog listing. There may be multiple spectra associated with any distinct catalog source. The spectroscopic library is a heterogeneous data set that includes low-resolution, to moderate-resolution, to high-resolution spectra, with wavelength coverage from the optical to the near-IR. The lowresolution and many of the moderate-resolution spectra generally result from QPQ campaigns focused on fast and efficient spectroscopic identification of photometric targets. A significant fraction of the optical, moderate-resolution spectra come from SDSS-LS or BOSS. The high-resolution spectra were specifically targeted toward, and have been used in detailed studies of, the CGM (QPQ3; QPQ9).

Over the years, related projects with broadened science goals have extended the QPQ catalog further. The various science cases have included measuring the small-scale clustering of quasars (Hennawi et al. 2006b, 2010; Myers et al. 2007, 2008; 


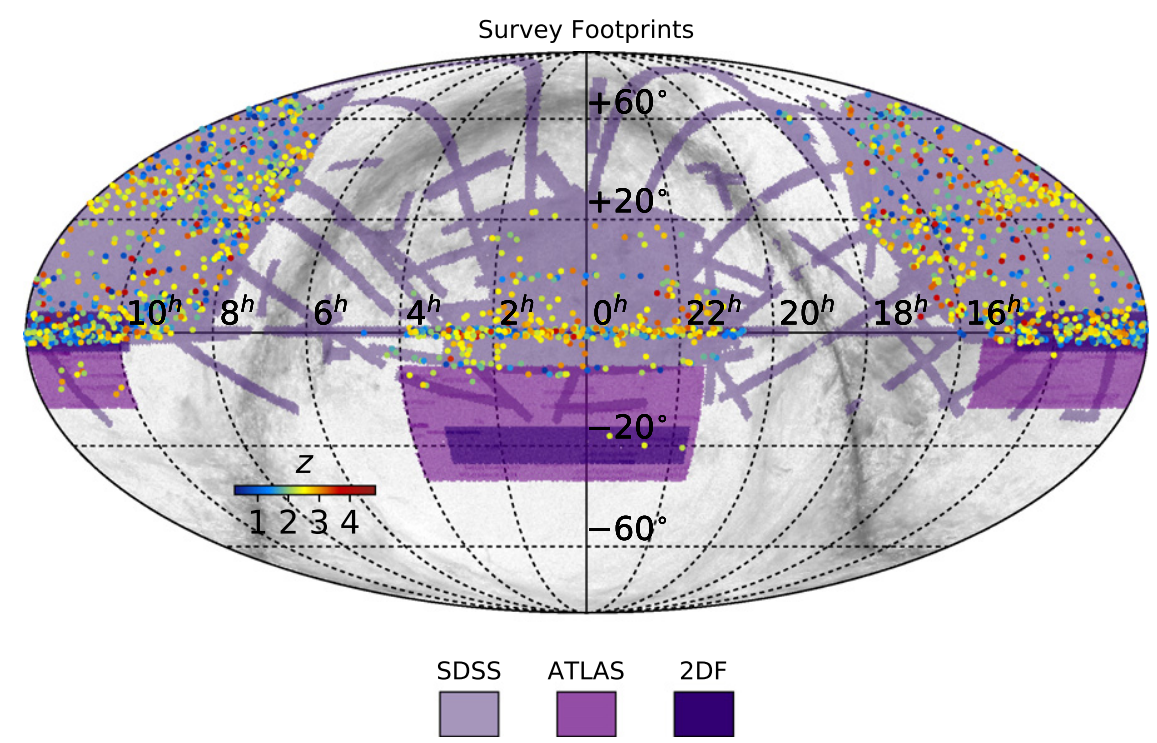

Figure 5. Sky coverage of the QPQ catalog in equatorial coordinates. The points show the locations of all foreground quasar pair members and are color-coded according to redshift. The shaded regions show the various survey footprints that bound the QPQ search area and are color-coded as given in the legend. The SDSS-LS and BOSS imaging footprints are plotted in the same color labeled "SDSS." The grayscale background shows the Milky Way polarized dust emission as seen by Planck.

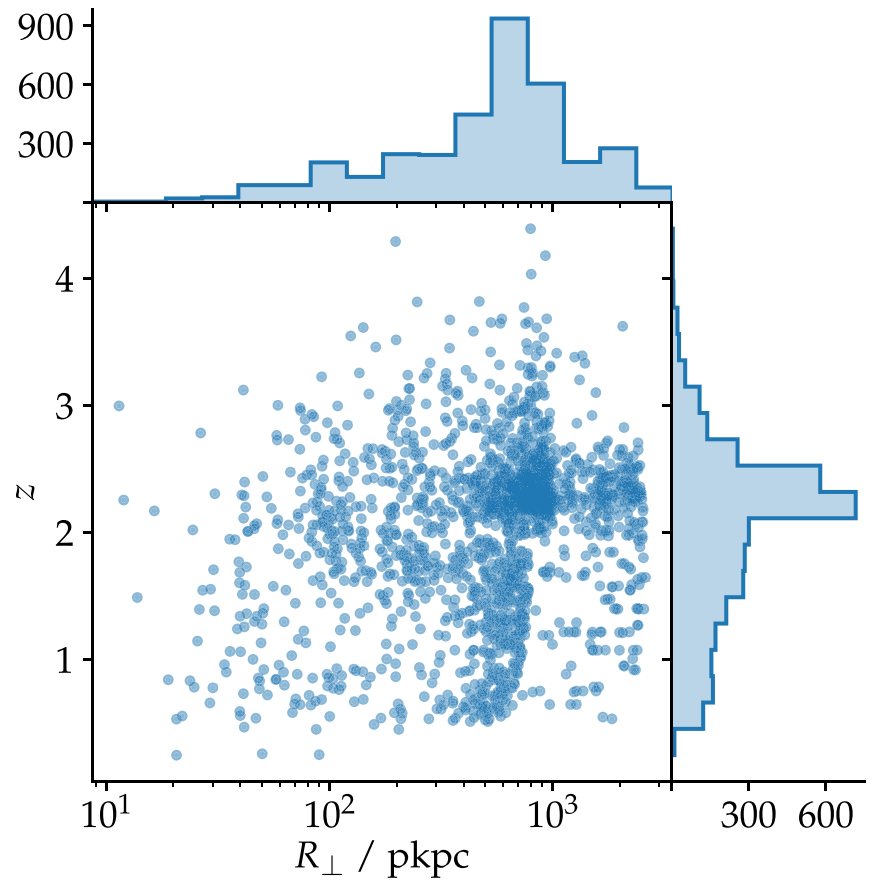

Figure 6. The joint distribution of redshift and physical transverse separation $R_{\perp}$ is shown in the main panel. Both are also plotted independently in the histograms in the peripheral panels. The peak in the redshift distribution corresponds to the peak in cosmic AGN activity. The distribution of physical transverse separation shows that the catalog probes the halos of quasars at impact parameters of tens of physical $\mathrm{kpc}$ to a few Mpc. Physical binaries are omitted in each of these plots by cutting the sample to pairs with velocity differences of $>3000 \mathrm{~km} \mathrm{~s}^{-1}$.

Shen et al. 2010; Eftekharzadeh et al. 2017), exploring correlations in the IGM along close-separation sightlines (Ellison et al. 2007; Martin et al. 2010), analyzing small-scale transverse Ly $\alpha$ forest correlations (Rorai et al. 2013), characterizing the transverse proximity effect (Schmidt et al. 2017), probing the halos of damped Ly $\alpha$ systems (DLAs; Rubin et al. 2015), and correcting CIV-based virial BH masses (Coatman et al. 2017)

The latter submission describes the near-infrared spectra of approximately 120 quasar pairs observed as part of QPQ follow-up programs. Coatman et al. also provide an additional 500 near-IR quasar spectra of non-pairs compiled both from the literature and from their own observations. At the time of writing all the Coatman et al. near-IR spectra are restricted to proprietary use but are expected to become publicly available in the very near future (P. C. Hewett 2018, private communication). As soon as this occurs, the near-IR pair spectra will be ingested by the QPQ database. ${ }^{16}$

The range in resolving power covered by the spectral library is shown for each instrument in Figure 7. The wavelength coverages of the spectra are characterized in Table 2, with respect to the optical and near-IR broadband filters of the SDSS and UKIDSS imaging surveys. Each row corresponds to a particular telescope and instrument in the catalog. The column "Total" refers to the total number of spectra. The columns ugrizYJHK refer to the SDSS or UKIDSS passbands of the same name and indicate the number of spectra with coverage in those passbands. A spectrum is arbitrarily considered to have coverage in a given passband when its wavelength array falls entirely or partially within the cut-on and cutoff wavelengths at $50 \%$ transmission. To avoid counting the less useful low signal-to-noise regions of any spectrum (usually found toward detector edges), positive wavelength coverage also requires that the average signal-to-noise in the 50 pixels on either side of the central covering pixel is at least 3 .

\subsection{Database Architecture}

The database comprises a catalog listing and a spectral library. The catalog listing is a simple table containing one record for each unique source in the database. Each field in the catalog is described in Table 3. The spectral library may

\footnotetext{
${ }^{16}$ Non-pair spectra will be ingested by the igmspec database, also under the SpecDB software package.
} 


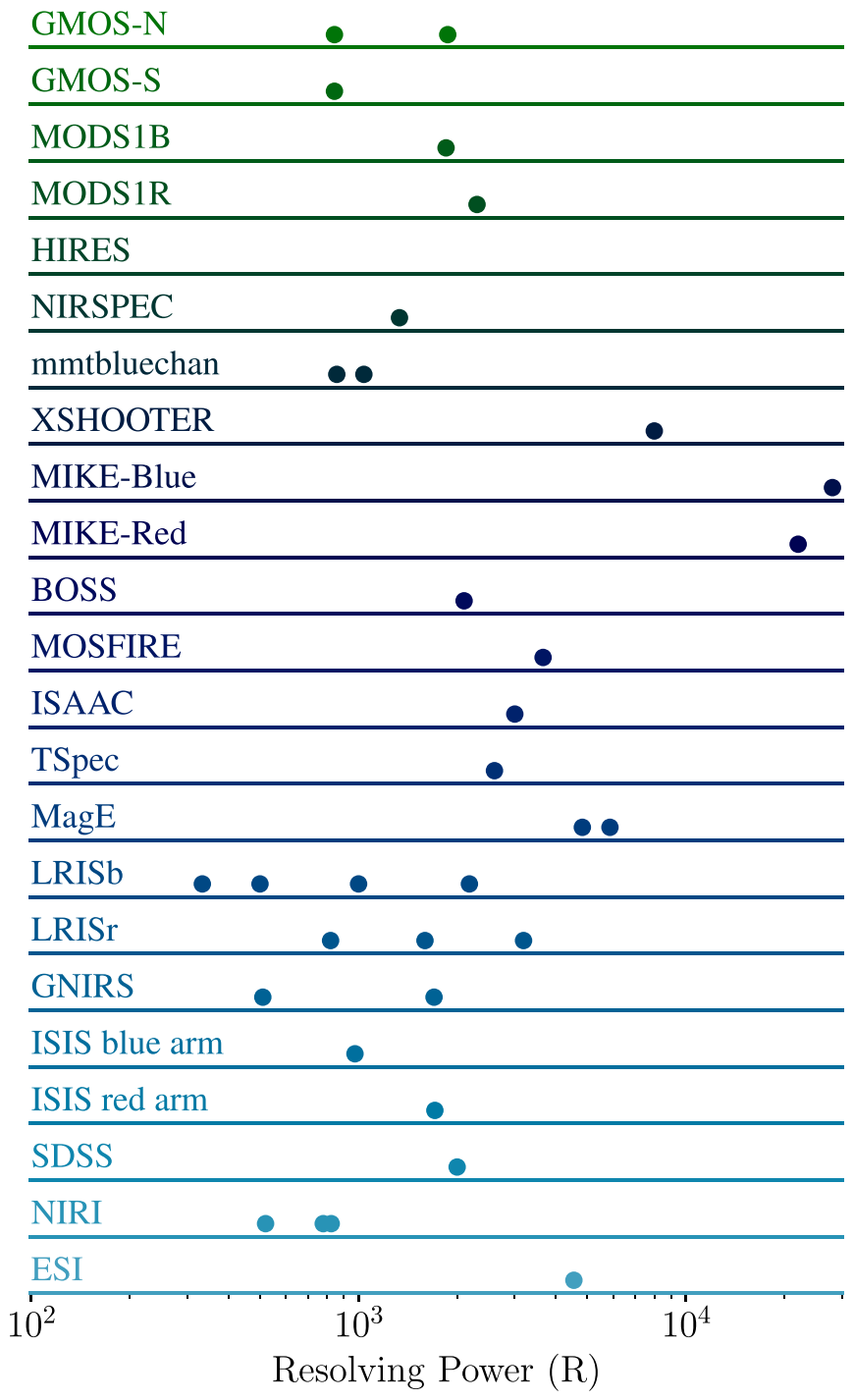

Figure 7. Range in spectral resolving power, grouped by instrument. Resolving power $(R)$ is given along the $x$-axis for each instrument listed on the $y$-axis. Note that no numerical scale is associated with the $y$-axis and that this figure does not give information pertaining to the number of spectra associated with a given instrument (see Table 2). Rather unique $R$-values for all spectra associated with a given instrument are represented by single points along the $x$-axis.

contain one or more spectra for each individual catalog source. A catalog record is linked to its corresponding spectra via a primary key field unique at the catalog level and has a one to many relationship with all spectra associated with that source.

Within the spectral library, spectra are arranged within a set of distinct groups. Each group contains the spectra and a metadata table, which maintains a list of the common properties pertaining to each spectrum, including the primary key, wavelength coverage, resolving power, telescope, and instrument, etc. Entries in the metadata table are ordered identically and aligned row-by-row with their corresponding spectra. A complete description of the metadata fields is given in Table 4.

The groups themselves are assigned and named according to the spectrograph used to measure the spectrum. A list of the groups can be found in Table 5. Note that in most cases the name of the group is identical to the corresponding value in the instrument metadata field. However, there are a few instances where this is not the case. In particular, where a set of observations on a single spectrograph results in a blue channel and a red channel spectrum and these spectra have not been merged, then two instruments exist inside a single group, one for the red channel and one for the blue channel.

Given this simple architecture it is straightforward to pull spectra out of the library through a catalog search. For example, one may query the catalog on any number of its fields to obtain a subsidiary table built to the query constraints. The primary key field of the subsidiary table can then be used to query the spectral library and retrieve the desired spectra. Of course, the catalog, spectral library, and metadata can also be queried independently of one another.

\section{Summary and Future Work}

With the addition here of 54 newly discovered quasar pairs from VST ATLAS, SDSS, and WISE, the QPQ database contains catalog listings for over 5500 distinct objects and a spectral database containing over 3500 optical and nearinfrared spectra of projected quasar pairs, quasars closely separated in redshift and gravitational lens candidates. The database is the fruit of over a decade of work, nine previously published articles, and many other related projects and studies. The projected pairs provide a means to probe the $z>2 \mathrm{CGM}$ of quasar host galaxies at impact parameters of tens of pkpc through to several $\mathrm{pMpc}$ or equivalently from scales comparable in extent to galactic disks, to bound gas in the CGM and to nearby regions of intergalactic space. In publishing this catalog the hope is to provide a laboratory for future discoveries in the CGM of massive galaxies hosting quasars. This database serves as a living resource that will continue to grow, reflecting advances in both scientific understanding and instrumentation.

New multi-fiber spectrographs such as DESI (Dark Energy Spectroscopic Instrument; DESI Collaboration et al. 2016) and Subaru PSF (Prime Focus Spectrograph; Tamura et al. 2016) will supply data sets for future catalog expansion. DESI alone will target and obtain redshifts for over $\sim 700,000$ quasars at $z \gtrsim 2$, providing gains of over 3 times in comparison to the combined SDSS, BOSS, and ongoing eBOSS quasar redshift surveys. The QPQ project lays the foundation for future pair searches in these data sets, as well as provides the techniques needed to study them in unprecedented and exquisite statistical detail.

The promise of future large spectroscopic surveys demands increasing numbers of parallel, detailed case studies. To that end, the pursuit of a much larger sample of high-resolution, high signal-noise spectra is of prime importance. The complexities of the CGM are manifest in its rich multiphase, multiscale structures, which display distinct kinematics and metallicities. Detailed dissection of all facets of the CGM requires the capability to resolve its smallest coherent structures. With current ground-based $10 \mathrm{~m}$ telescopes, few QPQ pairs are currently within range of echelle spectrographs that can provide resolutions of FWHM $\sim 10 \mathrm{~km} \mathrm{~s}^{-1}$. The arrival of $30 \mathrm{~m}$ class telescopes in the near future will place many of the QPQ pairs in the realm of these instruments and thus provide the required samples of high-resolution spectra.

High-resolution spectra are not useful for assessing the kinematics of distinct clouds or flows if line centroids cannot be measured with appreciable accuracy. This requires access to the H I Balmer series or narrow forbidden lines such as [O II] and [O III], which at $z \sim 2$ are redshifted into the near-IR. In order to refine current kinematic constraints and 
Table 2

Wavelength Coverage

\begin{tabular}{|c|c|c|c|c|c|c|c|c|c|c|c|}
\hline Telescope & Instrument & Total & $u$ & $g$ & $r$ & $i$ & $z$ & $Y$ & $J$ & $H$ & $K$ \\
\hline Gemini-North & GMOS-N & 71 & 28 & 70 & 37 & 0 & 0 & 0 & 0 & 0 & 0 \\
\hline Gemini-North & NIRI & 33 & 0 & 0 & 0 & 0 & 0 & 0 & 0 & 30 & \\
\hline Gemini-South & GMOS-S & 36 & 3 & 34 & 36 & 6 & 0 & 0 & 0 & 0 & \\
\hline MGIO-LBT & MODS1B & 10 & 7 & 10 & 0 & 0 & 0 & 0 & 0 & 0 & \\
\hline MGIO-LBT & MODS1R & 10 & 0 & 0 & 10 & 10 & 10 & 0 & 0 & 0 & \\
\hline Keck-I & LRISb & 358 & 258 & 216 & 129 & 0 & 0 & 0 & 0 & 0 & \\
\hline Keck-I & MOSFIRE & 2 & 0 & 0 & 0 & 0 & 0 & 0 & 1 & 2 & \\
\hline Keck-II & NIRSPEC & 5 & 0 & 0 & 0 & 0 & 0 & 0 & 0 & 2 & \\
\hline Keck-II & ESI & 115 & 0 & 86 & 109 & 112 & 108 & 89 & 0 & 0 & \\
\hline МMTO & mmtbluechan & 91 & 68 & 87 & 22 & 0 & 0 & 0 & 0 & 0 & \\
\hline ESO-VLT-U2 & XSHOOTER & 36 & 15 & 32 & 33 & 32 & 29 & 23 & 33 & 34 & \\
\hline ESO-VLT-U3 & ISAAC & 17 & 0 & 0 & 0 & 0 & 0 & 0 & 0 & 17 & \\
\hline SDSS $2.5-\mathrm{M}$ & BOSS & 2304 & 526 & 1896 & 1539 & 1526 & 1305 & 221 & 0 & 0 & 0 \\
\hline 200 & TSpec & 68 & 0 & 0 & 0 & 0 & 0 & 64 & 67 & 67 & 52 \\
\hline WHT & ISIS blue arm & 41 & 0 & 23 & 0 & 0 & 0 & 0 & 0 & 0 & 0 \\
\hline WHT & ISIS red arm & 41 & 0 & 0 & 35 & 29 & 0 & 0 & 0 & 0 & \\
\hline
\end{tabular}

Note. Wavelength coverage of individual spectra in the spectral library. Wavelength coverage is described with respect to the ugrizYJHK bandpasses of the SDSS and UKIDSS imaging surveys. A spectrum is arbitrarily defined to have coverage in a particular bandpass if its wavelength array falls within the interval of the cut-on/ cutoff values at $50 \%$ of the peak bandpass transmission. In order to avoid counting low signal-to-noise regions of the spectrum, the average signal-to-noise in the 50 pixels on either side of the central covering pixel must have a signal-to-noise of at least 3 . The column "Total" gives the total number of spectra grouped according to a particular telescope and instrument combination. The columns ugrizYJHK give the number of spectra deemed to have coverage in that bandpass.

Table 3

Catalog Schema

\begin{tabular}{|c|c|c|}
\hline$\overline{\mathrm{Key}}$ & Type & Description \\
\hline QPQ_ID & INT & Primary key unique identifier \\
\hline flag_group & INT & $\begin{array}{l}\text { Bitwise flag indicating the groups that the source } \\
\text { has spectra in }\end{array}$ \\
\hline zem & FLOAT & Emission redshift of source \\
\hline sig_zem & FLOAT & Estimated error in the redshift ${ }^{\mathrm{a}}$ \\
\hline flag_zem & STR & Key indicating source of the redshift ${ }^{\mathrm{b}}$ \\
\hline RA & FLOAT & Celestial R.A. in decimal degrees \\
\hline $\mathrm{DEC}$ & FLOAT & Celestial Decl. in decimal degrees \\
\hline flag_coo & STR & Key indicating source of the coordinates \\
\hline STYPE & STR & Spectral type (e.g., QSO) \\
\hline W1_FLUX & FLOAT & WISE W1 AB flux in nanomaggies \\
\hline W2_FLUX & FLOAT & WISE W2 AB flux in nanomaggies \\
\hline W3_FLUX & FLOAT & WISE W3 AB flux in nanomaggies \\
\hline W4_FLUX & FLOAT & WISE W4 $\mathrm{AB}$ flux in nanomaggies \\
\hline W1_IVAR & FLOAT & WISE W1 $\mathrm{AB}$ inverse variance in nanomaggies ${ }^{-2}$ \\
\hline W2_IVAR & FLOAT & WISE W2 $\mathrm{AB}$ inverse variance in nanomaggies ${ }^{-2}$ \\
\hline W3_IVAR & FLOAT & WISE W3 $\mathrm{AB}$ inverse variance in nanomaggies ${ }^{-2}$ \\
\hline W4_IVAR & FLOAT & WISE W4 $\mathrm{AB}$ inverse variance in nanomaggies ${ }^{-2}$ \\
\hline FUV_FLUX & FLOAT & GALEX FUV $\mathrm{AB}$ flux in nanomaggies \\
\hline NUV_FLUX & FLOAT & GALEX NUV AB flux in nanomaggies \\
\hline FUV_IVAR & FLOAT & $\begin{array}{l}\text { GALEX FUV AB inverse variance in } \\
\text { nanomaggies }^{-2}\end{array}$ \\
\hline NUV_IVAR & FLOAT & $\begin{array}{l}\text { GALEX NUV AB inverse variance in } \\
\text { nanomaggies }^{-2}\end{array}$ \\
\hline
\end{tabular}

Notes.

${ }^{\mathrm{a}}$ Redshift uncertainties are currently set to zero (see Section 6.1).

${ }^{\mathrm{b}}$ Possibilities are HW2010: Hewett \& Wild (2010), BOSS_PCA: Pâris et al. (2012), QPQ: QPQ1-QPQ1 and this submission.
Table 4

Metadata Schema

\begin{tabular}{lll}
\hline \hline Key & Type & Description \\
\hline QPQ_ID & INT & Primary key unique at the catalog level \\
GROUP_ID & INT & Primary key unique at the group level \\
IGM_ID & INT & Primary key reference into igmspec \\
zem_GROUP & FLOAT & Emission redshift \\
Sig_zem & FLOAT & Estimated error in the redshift \\
flag_zem & STR & Key indicating source of the redshift \\
RA_GROUP & FLOAT & R.A. in decimal degrees \\
DEC_GROUP & FLOAT & Decl. in decimal degrees \\
EPOCH & FLOAT & Year of epoch \\
R & FLOAT & Spectral resolution $(\delta \lambda / \lambda):$ FWHM \\
WV_MIN & FLOAT & Minimum wavelength value in $\AA$ \\
WV_MAX & FLOAT & Maximum wavelength value in $\AA$ \\
NPIX & INT & Number of pixels in the spectrum \\
SPEC_FILE & STR & Individual filename of the spectrum \\
STYPE & STR & Spectral type (e.g., QSO) \\
INSTR & STR & Instrument \\
DISPERSER & STR & Dispersing element \\
TELESCOPE & STR & Name of the telescope \\
GROUP & STR & Name of group \\
DATE-OBS & STR & Observation date \\
\hline
\end{tabular}

provide the foundation for future high-resolution observations, near-IR spectroscopy of quasar pairs is required. A campaign of near- IR spectroscopy has been undertaken as part of the QPQ project, the spectra themselves are released in the database presented here, precise redshift measurement from these spectra will be presented in a forthcoming paper 
Table 5

Groups within the Spectral Library

\begin{tabular}{lll}
\hline \hline & Group Names & \\
\hline GMOS & ISAAC & MODS \\
TRIPLESPEC & HIRES & MAGE \\
NIRSPEC & LRIS & MMT \\
GNIRS & XSHOOTER & SDSS \\
MIKE & NIRI & BOSS \\
ESI & MOSFIRE & ISIS \\
\hline
\end{tabular}

(H. F. Hennawi et al. 2018, in preparation), and near-IR spectroscopic follow-up of quasar pairs continues.

Compared to the successful cataloging of projected quasar pairs, the pursuit of galaxies at small impact parameters from bright quasar sightlines has been less fruitful. By the same tenet, these projections are required to probe the CGM of "normal" galaxies. Despite over a decade of searches on $10 \mathrm{~m}$ telescopes, only $\sim 10$ such sightlines currently exist with projected separations $\sim 200 \mathrm{pkpc}$. On the other hand, there is strong evidence to suggest that Lyman Limit Systems (LLS), which are easily detected in quasar absorption spectra, originate in galactic halos (e.g., Fumagalli et al. 2013, 2016). When LLSs are captured in the absorption spectra of two more closely separated quasars, one can use the LLS autocorrelation function along these multiple close sightlines to glean the extent, covering factor, and spatial profile of cool gas in the CGM.

It is similarly possible to study the interaction between different gas phases in the CGM by concentrating on intervening metal transitions. This experiment will elucidate the interplay between inflowing gas, expected to be metal-poor, and outflows, which will be enriched. Such experiments are well underway; QPQ-affiliated projects are using the $z \sim 3$ projected quasars presented in this submission and a sample of $z \sim 2$ quasar pairs, which have recently been observed by $H S T$ with the WFC3/UVIS grism, to study the correlation of LLSs across the epoch of peak galaxy formation.

In contrast to well-established techniques in absorption spectroscopy, the capacity to detect diffuse extragalactic gas in emission has been lacking until relatively recently. Cuttingedge techniques and advanced instruments have delivered some promising results and are set to alter this situation dramatically. Using custom built narrowband filters to image quasar fields, several authors have reported the presence of Enormous Ly $\alpha$ nebulae (ELAN) illuminated by elevated UV radiation fields (Cantalupo et al. 2014; Martin et al. 2014; Hennawi et al. 2015). Because of their projected angular sizes, ELAN are expected to extend well beyond the virial radii of quasar host galaxies, indicating that the emitting gas belongs to the surrounding IGM. This provides a new opportunity to study the characteristics of the gas feeding galaxies in emission and offers an independent and complementary probe to absorption studies. Integral field unit spectrographs such as MUSE (Multi Unit Spectroscopic Explorer Bacon et al. 2010), CWI (Cosmic Web Imager; Matuszewski et al. 2010), KCWI (Keck Cosmic Web Imager; Rockosi et al. 2016), and comparable instruments on $30 \mathrm{~m}$ class telescopes will begin to lead this field.

Hennawi et al. (2015) demonstrated that physical quasar pairs may be signposts of ELAN. They reported on the discovery of an ELAN in the presence of a physically associated quasar quartet. The chances of stumbling upon such a system serendipitously are $\sim 10^{-7}$, which strongly suggests a physical connection between ELAN and multiple quasars in overdense systems such as protoclusters. QPQ physical quasar pair fields provide ideal locations for current and future searches for ELAN.

J.R.F. and A.D.M. acknowledge support from NSF grants 1515404 and 1616168, NASA grant NNX16AN48G, and by the Director, Office of Science, Office of High Energy Physics of the U.S. Department of Energy under Contract No. DEAC02-05CH1123.

J.X.P. and M.W.L. acknowledge support from the National Science Foundation (NSF) grants AST-1010004 and AST1412981. M.F. acknowledges support by the Science and Technology Facilities Council grant number ST/P000541/1

The authors gratefully acknowledge the support that enabled observations on the WHT. The WHT is operated on the island of La Palma by the Isaac Newton Group of Telescopes in the Spanish Observatorio del Roque de los Muchachos of the Instituto de Astrofísica de Canarias. The WHT/ISIS spectroscopy was obtained as part of programs 2015/P1 and 2016/P6. We also acknowledge the support that enabled past observations at the Keck, Gemini, Large Binocular Telescope, Very Large Telescope, Las Campanas, MMT, Magellan, William Herschel Telescope, Palomar, and Apache Point Observatories

This work was based on data products from observations made with the ESO Telescopes at the La Silla Paranal Observatory under program ID 177.A-3011(A,B,C,D,E.F,G,H,I,J), We also acknowledge support from Science and Technology Facilities Council Consolidated Grant ST/P000541/1.

Funding for the SDSS, SDSS-II, and SDSS-III has been provided by the Alfred P. Sloan Foundation, the Participating Institutions, the National Science Foundation, the U.S. Department of Energy. SDSS and SDSS-II were additionally funded by the National Aeronautics and Space Administration, the Japanese Monbukagakusho, the Max Planck Society, and the Higher Education Funding Council for England. The SDSS website is http://www.sdss.org/ and the SDSS-III website is http://www.sdss3.org/.

The SDSS is managed by the Astrophysical Research Consortium for the Participating Institutions. The Participating Institutions are the American Museum of Natural History, Astrophysical Institute Potsdam, University of Basel, University of Cambridge, Case Western Reserve University, University of Chicago, Drexel University, Fermilab, the Institute for Advanced Study, the Japan Participation Group, Johns Hopkins University, the Joint Institute for Nuclear Astrophysics, the Kavli Institute for Particle Astrophysics and Cosmology, the Korean Scientist Group, the Chinese Academy of Sciences (LAMOST), Los Alamos National Laboratory, the Max-Planck-Institute for Astronomy (MPIA), the Max-PlanckInstitute for Astrophysics (MPA), New Mexico State University, Ohio State University, University of Pittsburgh, University of Portsmouth, Princeton University, the United States Naval Observatory, and the University of Washington.

SDSS-III is managed by the Astrophysical Research Consortium for the Participating Institutions of the SDSS-III Collaboration including the University of Arizona, the Brazilian Participation Group, Brookhaven National Laboratory, Carnegie Mellon University, University of Florida, the French Participation Group, the German Participation Group, Harvard University, the Instituto de Astrofisica de Canarias, the Michigan State/Notre Dame/JINA Participation Group, Johns 
Hopkins University, Lawrence Berkeley National Laboratory, Max Planck Institute for Astrophysics, Max Planck Institute for Extraterrestrial Physics, New Mexico State University, New York University, Ohio State University, Pennsylvania State University, University of Portsmouth, Princeton University, the Spanish Participation Group, University of Tokyo, University of Utah, Vanderbilt University, University of Virginia, University of Washington, and Yale University.

This publication makes use of data products from the Widefield Infrared Survey Explorer, which is a joint project of the University of California, Los Angeles, and the Jet Propulsion Laboratory/California Institute of Technology, funded by the National Aeronautics and Space Administration.

The 2dF QSO Redshift Survey (2QZ) was compiled by the 2QZ survey team from observations made with the $2^{\circ}$ Field on the Anglo-Australian Telescope.

Software: Numpy, Scipy, astropy, specDB, linetools, Pandas, SExtratcor, cmb_footprint, Topcat.

\section{ORCID iDs}

Joseph R. Findlay (i) https://orcid.org/0000-0002-3632-2015 J. Xavier Prochaska (ib https://orcid.org/0000-0002-7738-6875 Joseph F. Hennawi (i) https://orcid.org/0000-0002-7054-4332 Michele Fumagalli (i) https://orcid.org/0000-0001-6676-3842 Michael A. DiPompeo (1) https://orcid.org/0000-00016788-1701

Marie Wingyee Lau (다 https://orcid.org/0000-0001-9755-9406 Kate H. R. Rubin (1) https://orcid.org/0000-0001-6248-1864

\section{References}

Aihara, H., Allende Prieto, C., An, D., et al. 2011, ApJS, 193, 29 Bacon, R., Accardo, M., Adjali, L., et al. 2010, Proc. SPIE, 7735, 773508 Bertin, E., \& Arnouts, S. 1996, A\&AS, 117, 393

Blanton, M. R., Lin, H., Lupton, R. H., et al. 2003, AJ, 125, 2276

Bordoloi, R., Lilly, S. J., Knobel, C., et al. 2011, ApJ, 743, 10 Bordoloi, R., Tumlinson, J., Werk, J. K., et al. 2014, ApJ, 796, 136 Borthakur, S., Heckman, T., Tumlinson, J., et al. 2016, ApJ, 833, 259 Bouché, N., Finley, H., Schroetter, I., et al. 2016, ApJ, 820, 121 Bovy, J., Hennawi, J. F., Hogg, D. W., et al. 2011, ApJ, 729, 141 Bovy, J., Myers, A. D., Hennawi, J. F., et al. 2012, ApJ, 749, 41 Cantalupo, S., Arrigoni-Battaia, F., Prochaska, J. X., Hennawi, J. F., \& Madau, P. 2014, Natur, 506, 63

Chambers, K. C., Magnier, E. A., Metcalfe, N., et al. 2016, arXiv: 1612.05560

Chehade, B., Shanks, T., Findlay, J., et al. 2016, MNRAS, 459, 1179 Coatman, L., Hewett, P. C., Banerji, M., et al. 2017, MNRAS, 465, 2120 Croft, R. A. C. 2004, ApJ, 610, 642

Croom, S. M., Smith, R. J., Boyle, B. J., et al. 2004, MNRAS, 349, 1397 Dawson, K. S., Schlegel, D. J., Ahn, C. P., et al. 2013, AJ, 145, 10 DESI Collaboration, Aghamousa, A., Aguilar, J., et al. 2016, arXiv:1611.00036 DiPompeo, M. A., Bovy, J., Myers, A. D., \& Lang, D. 2015, MNRAS, 452, 3124 Doi, M., Tanaka, M., Fukugita, M., et al. 2010, AJ, 139, 1628

Eftekharzadeh, S., Myers, A. D., Hennawi, J. F., et al. 2017, MNRAS, 468, 77 Eftekharzadeh, S., Myers, A. D., White, M., et al. 2015, MNRAS, 453, 2779 Ellison, S. L., Hennawi, J. F., Martin, C. L., \& Sommer-Larsen, J. 2007, MNRAS, 378, 801

Faucher-Giguère, C.-A., Feldmann, R., Quataert, E., et al. 2016, MNRAS, 461, L32

Fernandez-Soto, A., Barcons, X., Carballo, R., \& Webb, J. K. 1995, MNRAS, 277, 235

Font-Ribera, A., Arnau, E., Miralda-Escudé, J., et al. 2013, JCAP, 5, 018 Fox, A. J., Bordoloi, R., Savage, B. D., et al. 2015, ApJL, 799, L7

Fox, A., \& Davé, R. (ed.) 2017, Gas Accretion onto Galaxies, Vol. 430 (Basel: Springer International Publishing AG)

Fukugita, M., Ichikawa, T., Gunn, J. E., et al. 1996, AJ, 111, 1748

Fumagalli, M., Cantalupo, S., Dekel, A., et al. 2016, MNRAS, 462, 1978

Fumagalli, M., Mackenzie, R., Trayford, J., et al. 2017, MNRAS, 471, 3686

Fumagalli, M., O'Meara, J. M., \& Prochaska, J. X. 2011, Sci, 334, 1245
Fumagalli, M., O’Meara, J. M., Prochaska, J. X., \& Worseck, G. 2013, ApJ, 775,78

Gunn, J. E., Siegmund, W. A., Mannery, E. J., et al. 2006, AJ, 131, 2332

Hennawi, J. F. 2004, PhD thesis, PhD dissertation, Princeton Univ.

Hennawi, J. F., Myers, A. D., Shen, Y., et al. 2010, ApJ, 719, 1672

Hennawi, J. F., \& Prochaska, J. X. 2007, ApJ, 655, 735

Hennawi, J. F., \& Prochaska, J. X. 2013, ApJ, 766, 58

Hennawi, J. F., Prochaska, J. X., Burles, S., et al. 2006a, ApJ, 651, 61

Hennawi, J. F., Prochaska, J. X., Cantalupo, S., \& Arrigoni-Battaia, F. 2015, Sci, 348, 779

Hennawi, J. F., Strauss, M. A., Oguri, M., et al. 2006b, AJ, 131, 1

Hewett, P. C., \& Wild, V. 2010, MNRAS, 405, 2302

Ho, S. H., Martin, C. L., Kacprzak, G. G., \& Churchill, C. W. 2017, ApJ, 835,267

Hogg, D. W., Finkbeiner, D. P., Schlegel, D. J., \& Gunn, J. E. 2001, AJ, 122, 2129

Johnson, S. D., Chen, H.-W., \& Mulchaey, J. S. 2015, MNRAS, 452, 2553

Jorden, P. R. 1990, Proc. SPIE, 1235, 790

Keeney, B. A., Stocke, J. T., Danforth, C. W., et al. 2017, ApJS, 230, 6

Kuijken, K. 2011, Msngr, 146, 8

Lang, D. 2014, AJ, 147, 108

Lang, D., Hogg, D. W., \& Schlegel, D. J. 2016, AJ, 151, 36

Lau, M. W., Prochaska, J. X., \& Hennawi, J. F. 2016, ApJS, 226, 25

Lau, M. W., Prochaska, J. X., \& Hennawi, J. F. 2017, arXiv:1705.03476

Lehner, N., \& Howk, J. C. 2011, Sci, 334, 955

Lewis, I. J., Cannon, R. D., Taylor, K., et al. 2002, MNRAS, 333, 279

Liske, J., \& Williger, G. M. 2001, MNRAS, 328, 653

Lupton, R., Gunn, J. E., Ivezić, Z., Knapp, G. R., \& Kent, S. 2001, in ASP Conf. Ser. 238, Astronomical Data Analysis Software and Systems X, ed.

F. R. Harnden, Jr., F. A. Primini, \& H. E. Payne (San Francisco, CA: ASP), 269

Mainzer, A., Bauer, J., Cutri, R. M., et al. 2014, ApJ, 792, 30

Mainzer, A., Bauer, J., Grav, T., et al. 2011, ApJ, 731, 53

Martin, C. L. 2005, ApJ, 621, 227

Martin, C. L., Scannapieco, E., Ellison, S. L., et al. 2010, ApJ, 721, 174

Martin, D. C., Chang, D., Matuszewski, M., et al. 2014, ApJ, 786, 106

Matuszewski, M., Chang, D., Crabill, R. M., et al. 2010, Proc. SPIE, 7735 , $77350 \mathrm{P}$

Meisner, A. M., Lang, D., \& Schlegel, D. J. 2017, AJ, 153, 38

Morrissey, P., Conrow, T., Barlow, T. A., et al. 2007, ApJS, 173, 682

Myers, A. D., Brunner, R. J., Richards, G. T., et al. 2007, ApJ, 658, 99

Myers, A. D., Palanque-Delabrouille, N., Prakash, A., et al. 2015, ApJS, 221, 27

Myers, A. D., Richards, G. T., Brunner, R. J., et al. 2008, ApJ, 678, 635

Neugebauer, G., Habing, H. J., van Duinen, R., et al. 1984, ApJL, 278, L1

Padmanabhan, N., Schlegel, D. J., Finkbeiner, D. P., et al. 2008, ApJ, 674,1217

Pâris, I., Petitjean, P., Aubourg, É., et al. 2012, A\&A, 548, A66

Pâris, I., Petitjean, P., Ross, N. P., et al. 2017, A\&A, 597, A79

Peeples, M. S., Werk, J. K., Tumlinson, J., et al. 2014, ApJ, 786, 54

Pettini, M., Kellogg, M., Steidel, C. C., et al. 1998, ApJ, 508, 539

Pier, J. R., Munn, J. A., Hindsley, R. B., et al. 2003, AJ, 125, 1559

Planck Collaboration, Ade, P. A. R., Aghanim, N., et al. 2015, A\&A, 576, A104

Planck Collaboration, Ade, P. A. R., Aghanim, N., et al. 2016, A\&A, 594, A13 Prochaska, J. X. 2017, A\&C, 19, 27

Prochaska, J. X., \& Hennawi, J. F. 2009, ApJ, 690, 1558

Prochaska, J. X., Hennawi, J. F., Lee, K.-G., et al. 2013a, ApJ, 776, 136

Prochaska, J. X., Hennawi, J. F., \& Simcoe, R. A. 2013b, ApJL, 762, L19

Prochaska, J. X., Lau, M. W., \& Hennawi, J. F. 2014, ApJ, 796, 140

Prochaska, J. X., Werk, J. K., Worseck, G., et al. 2017, ApJ, 837, 169

Putman, M. E., Peek, J. E. G., \& Joung, M. R. 2012, ARA\&A, 50, 491

Reid, B., Ho, S., Padmanabhan, N., et al. 2016, MNRAS, 455, 1553

Richards, G. T., Deo, R. P., Lacy, M., et al. 2009a, AJ, 137, 3884

Richards, G. T., Myers, A. D., Gray, A. G., et al. 2009b, ApJS, 180, 67

Richards, G. T., Myers, A. D., Peters, C. M., et al. 2015, ApJS, 219, 39

Richards, G. T., Nichol, R. C., Gray, A. G., et al. 2004, ApJS, 155, 257

Rockosi, C., Cowley, D., Cabak, J., Hilyard, D., \& Pfister, T. 2016, Proc. SPIE, 9908, 990854

Rorai, A., Hennawi, J. F., \& White, M. 2013, ApJ, 775, 81

Rosario, D. J., Trakhtenbrot, B., Lutz, D., et al. 2013, A\&A, 560, A72 Ross, N. P., Myers, A. D., Sheldon, E. S., et al. 2012, ApJS, 199, 3

Rubin, K. H. R., Hennawi, J. F., Prochaska, J. X., et al. 2015, ApJ, 808, 38

Rubin, K. H. R., Prochaska, J. X., Koo, D. C., et al. 2014, ApJ, 794, 156

Rubin, K. H. R., Prochaska, J. X., Koo, D. C., \& Phillips, A. C. 2012, ApJL, 747, L26 
Schipani, P., Capaccioli, M., Arcidiacono, C., et al. 2012, Proc. SPIE, 8444, $84441 \mathrm{C}$

Schirber, M., Miralda-Escudé, J., \& McDonald, P. 2004, ApJ, 610, 105

Schmidt, T. M., Worseck, G., Hennawi, J. F., Prochaska, J. X., \& Crighton, N. H. M. 2017, ApJ, 847, 81

Sembach, K. R., Wakker, B. P., Tripp, T. M., et al. 2004, ApJS, 150, 387

Shanks, T., Metcalfe, N., Chehade, B., et al. 2015, MNRAS, 451, 4238

Shen, Y., Brandt, W. N., Richards, G. T., et al. 2016, ApJ, 831, 7

Shen, Y., Hennawi, J. F., Shankar, F., et al. 2010, ApJ, 719, 1693

Smee, S. A., Gunn, J. E., Uomoto, A., et al. 2013, AJ, 146, 32

Smith, J. A., Tucker, D. L., Kent, S., et al. 2002, AJ, 123, 2121

Stanley, F., Alexander, D. M., Harrison, C. M., et al. 2017, MNRAS, 472,2221
Steidel, C. C., Erb, D. K., Shapley, A. E., et al. 2010, ApJ, 717, 289 Stoughton, C., Lupton, R. H., Bernardi, M., et al. 2002, AJ, 123, 485

Tamura, N., Takato, N., Shimono, A., et al. 2016, Proc. SPIE, 9908, 99081M

Thom, C., Tumlinson, J., Werk, J. K., et al. 2012, ApJL, 758, L41

Tumlinson, J., Peeples, M. S., \& Werk, J. K. 2017, ARA\&A, 55, 389

Tumlinson, J., Thom, C., Werk, J. K., et al. 2013, ApJ, 777, 59

Weiner, B. J., Coil, A. L., Prochaska, J. X., et al. 2009, ApJ, 692, 187

Werk, J. K., Prochaska, J. X., Thom, C., et al. 2013, ApJS, 204, 17

Wiseman, P., Perley, D. A., Schady, P., et al. 2017, arXiv:1705.01543

Wright, E. L., Eisenhardt, P. R. M., Mainzer, A. K., et al. 2010, AJ, 140, 1868

Wu, X.-B., Hao, G., Jia, Z., Zhang, Y., \& Peng, N. 2012, AJ, 144, 49

York, D. G., Adelman, J., Anderson, J. E., Jr., et al. 2000, AJ, 120, 1579 